\title{
Effects of timing and stability on laser wakefield acceleration using external injection
}

\author{
W. van Dijk, ${ }^{*}$ J. M. Corstens, S. B. van der Geer, ${ }^{\dagger}$ M. J. van der Wiel, and G. J. H. Brussaard \\ Center for Plasma Physics and Radiation Technology, Eindhoven University of Technology, POB 513, 5600 MB Eindhoven, \\ The Netherlands
}

(Received 11 February 2009; published 29 May 2009)

\begin{abstract}
The effects of experimental variations in the synchronization, laser power, and plasma density on the final beam parameters of externally injected electrons accelerated in a plasma wave are studied using a hybrid model. This model combines a relativistic fluid description of the plasma wave generated by the laser pulse with particle tracking of the accelerated electrons. For cases in which the effects of beam loading and laser depletion can be neglected, the two parts can be separated, allowing a significant reduction in computational power needed compared to particle in cell codes. Two different approaches to externally injecting electrons into plasma waves are studied: In the first case, the electrons are injected behind a laser pulse with $a_{0}=0.32$. In the second case, electrons are injected in front of the laser pulse in three different laser regimes $a_{0}=0.32, a_{0}=0.56$, and $a_{0}=1.02$, ranging from linear to nonlinear. For these four cases, the effects of expected experimental variations in synchronization ( $\pm 500 \mathrm{fs}$ ), laser power $( \pm 10 \%)$, and plasma density $( \pm 30 \%)$ are studied. From these simulations, it becomes clear that in some cases, even a small variation in one of these parameters can create a large change in the final energy, energy spread, and trapped charge. For lower laser intensities, the method of injecting behind the laser pulse is the least sensitive to fluctuations while injection in front of the laser pulse becomes less sensitive at higher intensities.
\end{abstract}

DOI: 10.1103/PhysRevSTAB.12.051304

PACS numbers: 41.75.Jv, 52.38.Kd, 41.85.Ar, 52.65.Ww

\section{INTRODUCTION}

Laser wakefield acceleration [1] allows the acceleration of electrons in electric fields orders of magnitude larger than those achieved in conventional accelerators. Already, experiments have shown the ability to create electron bunches by laser wakefields with energies of $100 \mathrm{MeV}$ [2-4] and even up to $1 \mathrm{GeV}$ [5] with low energy spread.

Currently, one of the major areas of interest is the reproducibility of the beams created in these acceleration experiments. Since these experiments rely on highly nonlinear plasma waves to trap electrons from the plasma (wave breaking) and accelerate them, they are sensitive to variations in laser power and plasma density. To improve reproducibility, one needs to improve control of the injection by using, for example, optical injection [6], plasma density gradients [7], or an external source of electrons [8-11].

In this paper, we focus on the use of external injection. In order to evaluate the reproducibility of this scheme, we will use a numerical model to investigate the effect of variations in the laser power and plasma density. Since the use of external injection also requires synchronization between the electron source and the laser pulse used to excite the

\footnotetext{
*w.v.dijk@tue.nl

${ }^{\dagger}$ Also at Pulsar Physics, Burghstraat 47, 5614 BC Eindhoven, The Netherlands. http://www.pulsar.nl
}

plasmas wave, we will also look at the effect of variations in this synchronization.

\section{MODEL}

\section{A. Framework}

In laser wakefield acceleration simulations, particle in cell (PIC) codes [12-14] are commonly used to calculate the time evolution of both electron bunch and plasma. However, when long plasmas are used, the computational power needed for these simulations increases very rapidly. This becomes problematic when multiple simulation runs are desired. In our previous work $[11,15]$, a combination of the particle tracking program GPT [16] and an analytical description of linear wakefields using simple harmonics was used to greatly reduce the computation time needed. This allowed the evaluation of a large number of different parameter settings and the use of long plasma channels. However, this approach is no longer valid when the laser creating the plasma wave becomes powerful enough to generate nonlinear waves or when including the effect of radial plasma gradients. These radial plasma gradients are used for guiding the laser through the plasma to prevent it from defocusing and thus to retain the high intensity of the focused laser pulse [17-20].

To accommodate these effects, we now employ a relativistic fluid model (described in Sec. II B) to describe the plasma and the plasma wave. The fields calculated with 
this fluid model are then implemented in the particle tracking code in order to obtain their effect on the electron bunch.

\section{B. Fluid model}

In order to describe the plasma wave, we use a system of relativistic hydrodynamic equations of motion for the plasma electrons, the Maxwell equations, and a beam envelope description of the laser pulse in an approach analogous to [21,22]. This approach results in the following set of equations for the plasma evolution:

$$
\begin{gathered}
\frac{\partial n}{\partial t}+\vec{\nabla} \cdot(n \vec{v})=0, \\
\frac{\partial \vec{p}}{\partial t}=e \vec{E}-m_{0} c^{2} \vec{\nabla} \gamma, \\
\frac{\partial \vec{E}}{\partial t}=-4 \pi e n \vec{v}-\frac{c^{2}}{e} \vec{\nabla} \times(\vec{\nabla} \times \vec{p}), \\
\gamma=\sqrt{1+\frac{|\vec{p}|^{2}}{m_{0}^{2} c^{2}}+\frac{|\vec{a}|^{2}}{2}}, \\
\vec{v}=\frac{\vec{p}}{m_{0} \gamma} .
\end{gathered}
$$

Here $e$ is the charge of an electron, $m_{0}$ the electron rest mass, and $c$ the speed of light. The plasma electrons are described by their density $n$, momentum $\vec{p}$, velocity $\vec{v}$, and Lorentz factor $\gamma$. The electric field strength is denoted by $\vec{E}$ and the normalized vector potential of the laser by $\vec{a}$ :

$$
\vec{a}=\frac{e \vec{A}}{m_{0} c}
$$

with $\vec{A}$ the vector potential of the laser pulse.

\section{Moving fields}

The equations given in Sec. II B can describe the wakefields generated by a laser pulse for any given position and time as long as the amplitude of the plasma wave stays (well) below the limit of wave breaking. Incorporating them directly into a particle tracking code will however

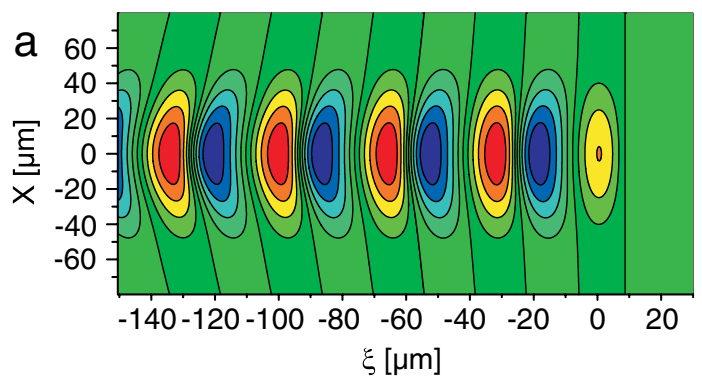

mean that the evolution of the plasma has to be evaluated for the entire plasma during each time step. This again requires a large amount of computer power for larger plasmas.

To reduce the amount of computational power needed, we have made a few assumptions about the different interactions: If the total charge (density) in the injected electron bunch is low enough, the effect of the electron bunch on the plasma can be neglected (see the Appendix for detailed analysis) which allows the decoupling of the plasma and the electron bunch evolution. If we further assume the presence of a parabolic plasma profile matched to the laser pulse for guiding, laser beam divergence is absent. Last, we will assume the effect of the plasma on the laser pulse (laser frequency/envelope evolution and laser depletion) can be neglected. With these assumptions, the plasma evolution becomes time independent in the frame of the laser pulse. This also means that only the plasma in the region of interest, i.e., close to the laser pulse has to be evaluated and the rest of the plasma can be ignored in the plasma evolution. Thus, the amount of calculations is only dependent on the size of the region of interest and not on the total length of the plasma the laser pulse is traveling through.

To calculate the plasma wave in a moving frame, we evaluate the equations in the coordinates moving at the same velocity as the laser pulse, $\xi$ :

$$
\xi=z-v_{g} t
$$

The group velocity $v_{g}$ of the laser pulse is described by

$$
v_{g}=c \sqrt{1-\frac{n_{p}}{n_{\mathrm{cr}}}},
$$

with $n_{\text {cr }}$ the critical plasma density $\left(n_{\mathrm{cr}}=\frac{\epsilon_{0} m_{0}}{e^{2}} \omega_{l}^{2}\right)$ for angular frequency $\omega_{l}$ of the laser that is used to generate the wakefield. In this moving frame, the normalized laser vector potential $\vec{a}$ is described by

$$
\vec{a}(\xi, r)=a_{0} \exp \left[-\left(\frac{\xi-\xi_{0}}{\sigma_{t}}\right)^{2}-\left(\frac{r}{\sigma_{r}}\right)^{2}\right] \hat{e}_{z},
$$

with $a_{0}$ the potential maximum (calculated from the laser parameters), $\sigma_{t}$ the temporal, and $\sigma_{r}$ the radial extension

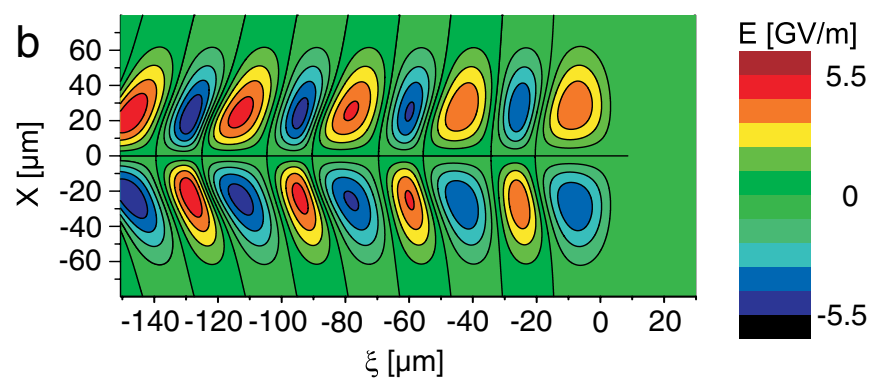

FIG. 1. (Color) Electric fields in the longitudinal (a) and radial (b) direction of a plasma wave in a plasma with $n_{e}=1.0 \times 10^{24} \mathrm{~m}^{-3}$ generated with laser pulse of $a_{0}=1.02$ (100 TW during $50 \mathrm{fs}$ matched to the matched spot size of $51.6 \mu \mathrm{m}$ ). The magnetic fields are not shown. 
of the laser pulse. The potential maximum $a_{0}$ can be calculated from the laser wavelength $\lambda_{l}$ and laser peak intensity $I_{\text {peak }}$ by

$$
a_{0} \simeq 0.85 \times 10^{-10} \lambda_{l}[\mathrm{~nm}] \sqrt{I_{\text {peak }\left[\frac{W}{m^{-2}}\right]}} .
$$

These equations and those of the plasma evolution [Eqs. (1)-(5)], are used to numerically calculate the electric and magnetic fields of the plasma wave in the moving frame. These fields are then inserted in the GPT particle tracking code to calculate their effect on electron bunches injected from an external source. A typical example of the electric fields calculated by this model is shown in Fig. 1.

If the electric and magnetic fields expel an electron beyond the physical radial boundaries of the plasma (the wall), the electrons are removed from the simulation.

\section{BASIC PARAMETERS}

\section{A. Electron bunch parameters}

The properties of the electron bunches injected into the plasma will be kept the same as much as possible in the different cases studied. Only the injection energy of the electrons will be changed for the different regimes to maximize the amount of trapped charge and final energy while minimizing the energy spread. Since the effect of bunch charge on the plasma is neglected, we assume a relatively low initial bunch charge of $10 \mathrm{pC}$. Also the bunches should be both focused and short to improve trapping. The electron bunch properties have been summarized in Table I.

\section{B. Plasma parameters}

The model for the plasma is based on a slow capillary discharge plasma [23] with a on-axis density of $1.0 \times$ $10^{24} \mathrm{~m}^{-3}$ and a radius of $150 \mu \mathrm{m}$. In order to get the matched spot size [24] for such a channel, we use the numerical formula given in [25], which leads to a matched spot size of $51.6 \mu \mathrm{m}$. The length of the plasma in the model is optimized for the different injection schemes to obtain a maximum final energy and minimum energy spread.

\section{RESULTS}

\section{A. Injection approach}

When externally injecting electrons there are two different approaches: electrons can be injected behind the laser pulse [11] or in front of the laser pulse [9]. For the present

TABLE I. General parameters of injected electron bunches.

\begin{tabular}{lr}
\hline \hline Charge [pC] & 10 \\
Radial size (FWHM) [ $\mu \mathrm{m}]$ & 30 \\
Length (FWHM) [fs] & 150 \\
\hline \hline
\end{tabular}

purpose, we will first look at the effects of timing jitter and variations in the laser energy and plasma density when injecting behind the laser pulse (Sec. IV B) and then compare those to the effects for injection in front of the laser pulse (Sec. IV C).

\section{B. Injection behind the laser pulse}

External injection of electrons behind the laser pulse offers several advantages compared to self-injection: It only requires the use of "modest" power laser systems [11] and can be used in combination with lower density plasmas [15] while still producing electron beams with low energy spread [8].

For the current study, we will assume a system that injects an electron bunch $300 \mathrm{fs}$ after the laser pulse into the plasma channel. The injection moment is chosen as a compromise between the effects of plasma dampening and possible electron bunch jitter: In order to prevent dampening of the plasma wave, it is best to inject as close as possible after the laser pulse. Injection in front of the laser pulse due to synchronization jitter can be minimized by injecting further behind the laser pulse. The laser wavelength is assumed to be $800 \mathrm{~nm}$, with a $10 \mathrm{TW}$ pulse of 50 fs FWHM temporal duration. This yields a normalized laser vector potential $a_{0}$ of 0.32 . We still have two remaining free parameters: injection energy and plasma length. To determine these, we first optimize the injection energy while striving for maximum energy, minimum energy spread, and maximum trapping: If the injection energy is too low, few or no electrons get trapped by the plasma wave. Too high an injection energy leads to a high energy spread and lower final energy. The optimum is thus found just above the trapping limit for most of the electrons. This yields an injection energy of $4.09 \mathrm{MeV}$. The plasma length is chosen equal to the position in the plasma where this optimum is achieved, which in this case is $30.0 \mathrm{~mm}$. The settings have been summarized in Table II.

The resulting energy spectrum of the accelerated electrons is shown in Fig. 2. When evaluating the effect of fluctuations in timing, laser energy, and plasma density, we will look at the relative changes of three main beam characteristics of the accelerated beam: energy, energy spread, and the number of trapped electrons relative to the number of injected electrons. As a reference, the values of these characteristics are given in Table III. Note that

TABLE II. Initial parameters of electron bunches injected behind the laser pulse.

\begin{tabular}{lc}
\hline \hline Laser wavelength [nm] & 800 \\
Laser power @50 fs [TW] & 10 \\
Laser vector potential $\left(a_{0}\right)[-]$ & 0.32 \\
Moment of injection for electrons [fs] & +300 \\
Electron kinetic energy [MeV] & 4.09 \\
Plasma length [mm] & 30.0 \\
\hline \hline
\end{tabular}




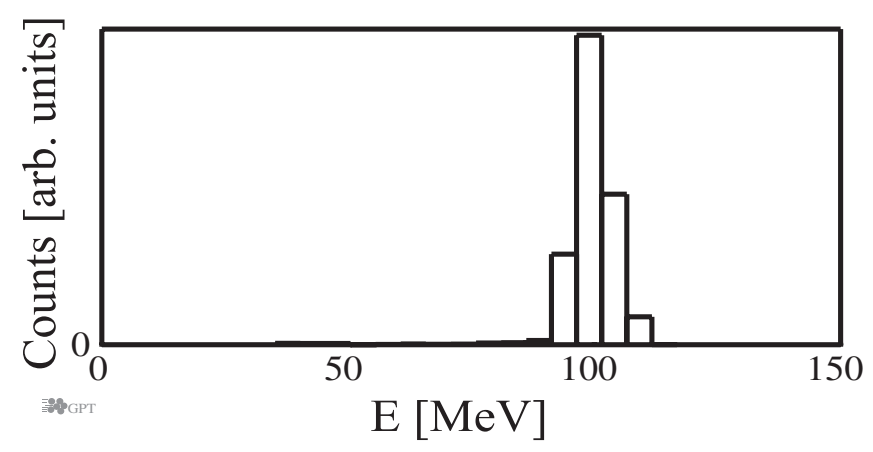

FIG. 2. Energy spectrum of the accelerated bunch at design settings (Table II) for $a_{0}=0.32$ with injection $300 \mathrm{fs}$ behind the laser pulse.

these are all single bunch characteristics, the effects of multiple bunches is discussed in VA.

\section{Bunch evolution for injection behind the laser pulse}

Before looking at the effect of the parameter fluctuations on the final bunch properties, we will first look at the normal evolution of the electron bunch at its design settings. This will help to better understand the underlying mechanisms as well as act as a framework for understanding the changes due to fluctuations.

The typical evolution for an optimized electron bunch injected after the laser pulse $[8,11]$ is shown in Fig. 3.

At $t=0$, the electron bunch with Gaussian radial and temporal profiles and a uniform energy is just in front of the plasma. At $t=8 \mathrm{ps}$, the electron bunch has entered the plasma and starts interacting with the plasma wave created by the laser pulse that entered the plasma 300 fs before the electrons. Since the electron bunch is several plasma wavelengths long, they are injected over all the phases of the plasma wave. Depending on the local phase of the plasma wave (see Fig. 1), the electrons are accelerated or decelerated and focused or expelled. At $t=15 \mathrm{ps,} \mathrm{those} \mathrm{elec-}$ trons in the defocusing phase of the plasma wave are starting to get expelled. Electrons in the focusing and accelerating phase continue to gain energy. At $t=60 \mathrm{ps,}$ most electrons in the defocusing phases have been radially expelled and removed from the simulation, while those that have been trapped continue to gain energy. The alternating focusing and defocusing fields in the plasma have now split the initial electron bunch into several sub-bunches all separated by one plasma wave period. At $t=105 \mathrm{ps}$, the electron bunch has just exited the plasma. As mentioned

TABLE III. Final properties of the electron bunch injected 300 fs behind the laser pulse.

\begin{tabular}{lr}
\hline \hline Laser vector potential $\left(a_{0}\right)[]$ & 0.32 \\
Average electron energy $[\mathrm{MeV}]$ & 100 \\
Electron energy spread $[\mathrm{MeV}]$ & 3 \\
Electron fraction trapped $[\%]$ & 19 \\
\hline \hline
\end{tabular}

before, the length of the plasma channel has been chosen such that most of the electrons are dephased, i.e., they are between the accelerating and decelerating phase of the plasma wave at the end of the plasma. This results in a maximum average energy and minimum energy spread.

\section{Timing}

First we will look at the effect of fluctuations in the timing between the laser pulse and the electrons. Since the plasma is typically fully ionized and guiding for tens or hundreds of nanoseconds [23,26] and the duration of the electron bunch and laser pulse are on the order of tens to a few hundred femtoseconds, the timing with respect to the plasma is less of a concern. The main concern therefore is the effect of timing fluctuations between the laser pulse and the electron bunch. Synchronization on the (tens of) femtosecond scale is experimentally quite challenging. The fluctuations of the electron bunch with respect to the laser can be as low as 100 fs [27], but are typically larger. For the present purpose, we will therefore assume a maximum fluctuation in timing or jitter of $500 \mathrm{fs}$ from the design point (300 fs behind the laser pulse). This means the electrons can also be injected in front of the laser pulse. The simulated effects of the timing variation $(\delta t)$ can be seen in Fig. 4.

As can be seen in Fig. 4, the final energy of the electrons seems almost insensitive to laser-electron synchronization jitter. This is to be expected since the strength of the accelerating fields for the trapped electrons remains almost constant and the acceleration length is the same for all electrons injected behind the laser pulse (those injected in front of the laser pulse do not get trapped). The trapped charge increases as the electrons are injected later into the plasma. In this simulation, where only the timing changes, the number of electrons trapped from a bunch injected behind the laser pulse is largely determined by the overlap within the plasma wave of the longitudinal accelerating and radial focusing fields. This overlap of the accelerating and focusing phase of the plasma wave changes as one moves further behind the laser pulse due to the radial plasma profile present in the plasma channel: Since the local wave velocity is dependent on the local plasma density [see Eq. (8)], there is also a radial gradient in the wave velocity of the plasma wave. This gradient changes the radial curvature of the plasma wave, increasing the overlap between the accelerating and focusing phases in the plasma wave in time as can be seen on the left side of Fig. 1. This increased overlap means larger parts of the plasma wave are both accelerating and focusing leading to more trapping. Since the accelerating fields in these extra parts are (slightly) different from those that are trapping and accelerating close to the laser pulse, this also leads to an increase in energy spread. When the electrons are injected closer to the laser pulse $(\delta t>0)$, part of the electron bunch will start to overlap with the laser pulse 

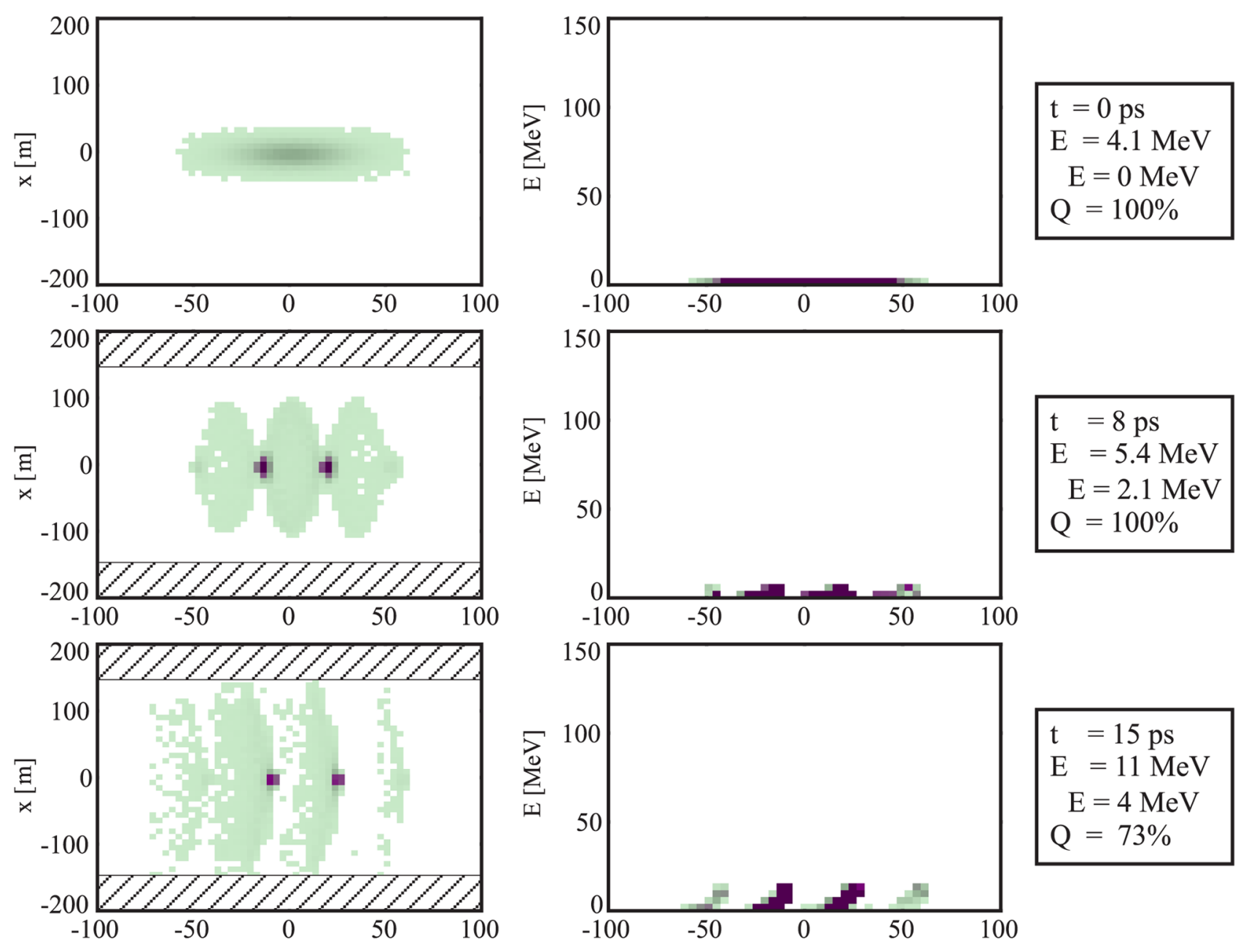

$$
\begin{aligned}
\mathrm{t} & =8 \mathrm{ps} \\
\mathrm{E} & =5.4 \mathrm{MeV} \\
\mathrm{E} & =2.1 \mathrm{MeV} \\
\mathrm{Q} & =100 \%
\end{aligned}
$$
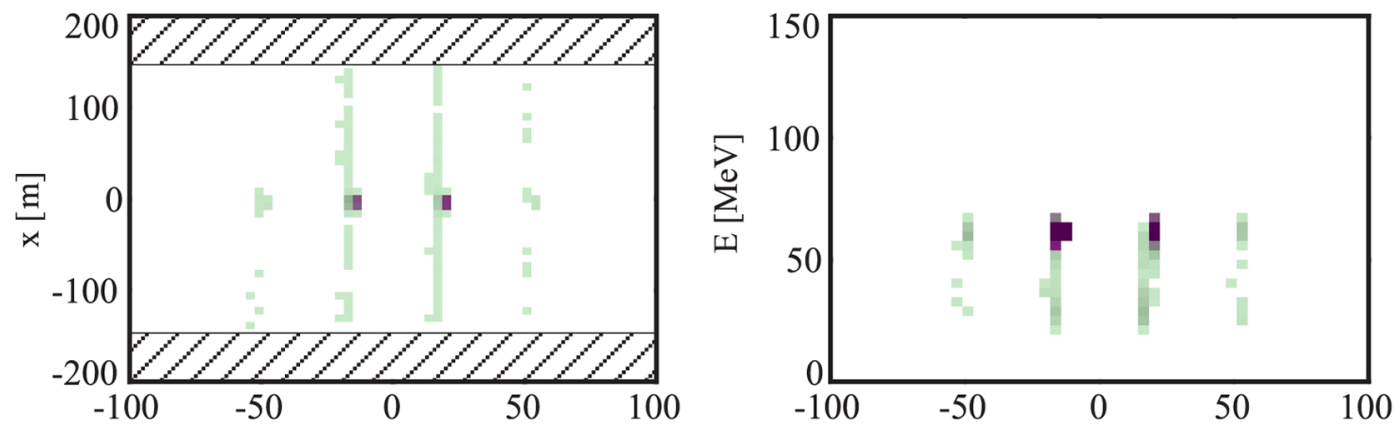
$\mathrm{t}=15 \mathrm{ps}$
$\mathrm{E}=11 \mathrm{MeV}$
$\mathrm{E}=4 \mathrm{MeV}$
$\mathrm{Q}=73 \%$
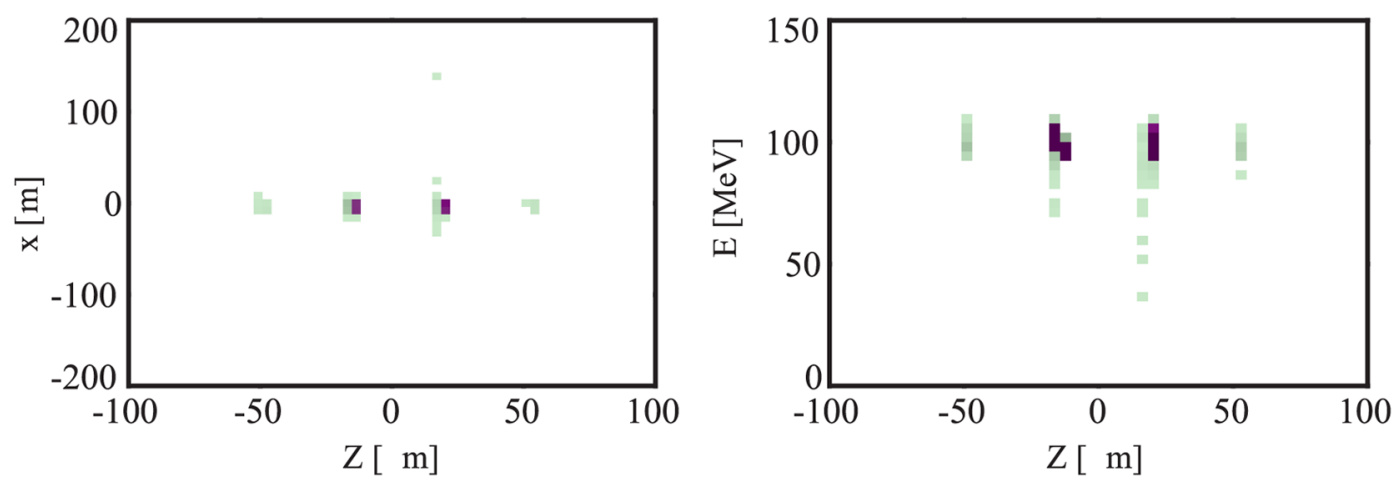

$\begin{aligned} \mathrm{t} & =60 \mathrm{ps} \\ \mathrm{E} & =61 \mathrm{MeV} \\ \mathrm{E} & =8 \mathrm{MeV} \\ \mathrm{Q} & =20 \%\end{aligned}$

$\mathrm{t}=105 \mathrm{ps}$

$\mathrm{E}=100 \mathrm{MeV}$

$\mathrm{E}=5 \mathrm{MeV}$

$\mathrm{Q}=19 \%$

FIG. 3. (Color) Density plot of the time evolution for the radial profile (left) and energy (right) of an electron bunch injected into a plasma wave from a laser pulse with $a_{0}=0.32$. For each snapshot, the time $(t)$, energy $(E)$, energy $\operatorname{spread}(\sigma E)$, and charge $(Q)$ are given on the right. 


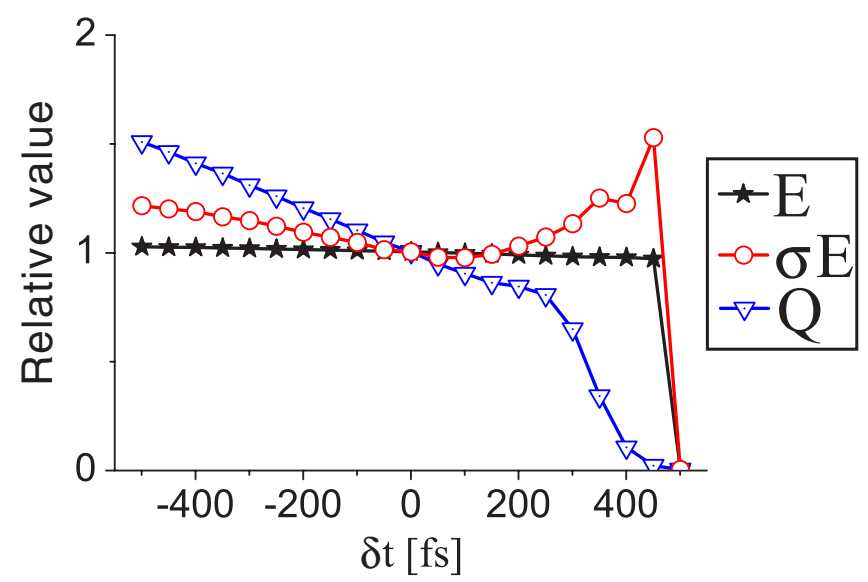

FIG. 4. (Color) Effect of \pm 500 fs fluctuations in the timing between laser and electrons $(\delta t>0$ means the electrons are injected further in front of the laser pulse) on the final beam parameters [energy $(E)$, energy spread $(\sigma E)$, and trapped fraction $(Q)$ ] for injection 300 fs behind the laser pulse with $a_{0}=0.32$.

and even enter the plasma before the laser. Since the energy of the electrons is too low to be trapped when injected in front of the laser pulse (see Sec. IV C), those electrons are expelled and fewer electrons get trapped. This continues until the entire electron bunch is radially expelled at $\delta t=$ $+500 \mathrm{fs}$. Between $\delta t=200 \mathrm{fs}$ and $\delta t=400 \mathrm{fs}$, some of these expelled electrons have not reached the point where they are removed when exiting the plasma. This increases the calculated energy spread.

\section{Laser energy variations}

Second, we will look at the effects of laser pulse energy. High power pulsed laser systems generally suffer from larger fluctuations in laser (pulse) energy compared to lower power systems. These fluctuations in laser power vary from system to system. We will consider fluctuations up to $10 \%$ from the nominal energy and look at the effects of these fluctuations. A laser pulse with a lower intensity will generate a plasma wave with lower amplitude which is expected to lead to lower final energy. Since a smaller part of the plasma wave is capable of trapping the electrons, one expects a lower trapped charge and lower energy spread. The reverse would hold for laser pulses with a higher intensity. The simulation results for laser energy fluctuations are shown in Fig. 5.

In Fig. 5 we recognize the expected trends. The final energy scales almost linearly with the laser energy though only weakly as expected from linear theory $[15,28]$. The increase and decrease of the trapped charge and energy spread depend more strongly on the laser energy. A more powerful laser pulse will generate a plasma wave with a larger amplitude which will trap electrons over a larger phase and thus a greater variety of final energies.

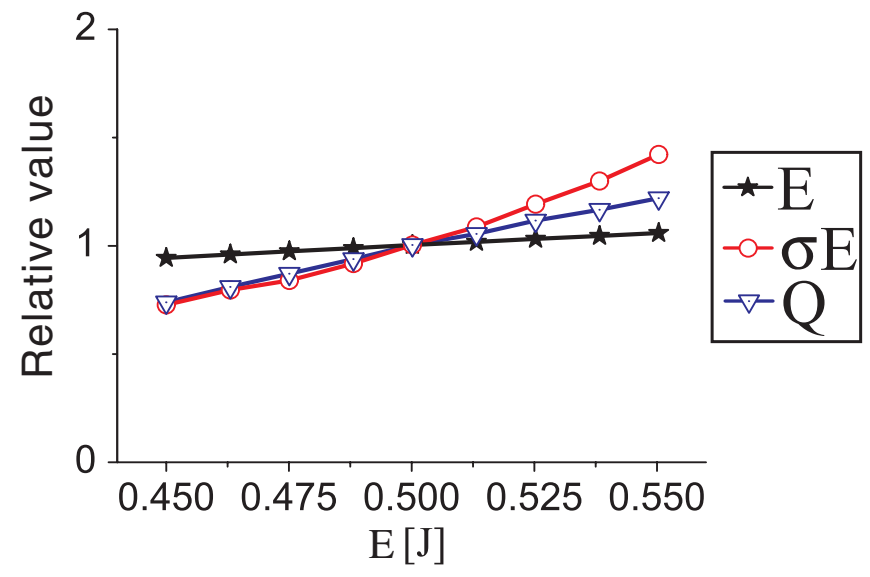

FIG. 5. (Color) Effect of $\pm 10 \%$ fluctuations in the laser energy on the final beam parameters [energy $(E)$, energy spread $(\sigma E)$, and trapped fraction $(Q)$ ] for a laser of $a_{0}=0.32$ with injection 300 fs behind the laser pulse.

\section{Density}

Third, we will look at the effect of plasma density fluctuations. The plasma density will depend on the initial gas pressure in the channel and the amount of energy deposited into the plasma during the discharge. Both these values can be hard to control in an experiment, especially when operating at discharge frequencies of 1-10 Hz. Measurements on the plasma density in a capillary discharge plasma performed by Gonsalves et al. [29] show an estimated measurement error/fluctuation in the plasma density of $20 \%$ with a maximum of about $30 \%$. We will therefore assume a maximum variation in the plasma density of $30 \%$. The effects of variations in the plasma density are harder to predict since there are two distinct effects that will influence the final bunch properties: First, a higher plasma density will lead to stronger fields in the plasma wave for a given laser energy. This will lead to higher energy, higher energy spread, and more trapped electrons. Second, an increased plasma density will shorten the dephasing length of the electrons. First of all, this means that the time that the electrons are in the accelerating phase is shorter, leading to a lower final energy. Second, the electrons will travel beyond the dephasing length before they leave the plasma channel entering the decelerating phase, this leads to a lower final energy and a higher energy spread. Third, since the electrons spend less time in the accelerating phase, they also slip into the defocusing phase faster since they are accelerated to a lower energy, this decreases the amount of trapped charge. For lower plasma densities, the dephasing length will increase which means the electrons will not have reached dephasing yet and are still being accelerated when reaching the end of the plasma channel leading to a higher final energy, higher energy spread. Also the amount of trapped electrons is expected to increase since they can be accelerated longer before entering the defocusing phase of the plasma wave. The 
TABLE IV. Expected effect of the variations in plasma density due to both the changing plasma wave field strength $(\Delta \vec{E})$ and the changed dephasing length $\left(\Delta \lambda_{\text {deph }}\right)$ on the energy $(E)$, energy spread $(\sigma E)$, and trapped charge $\left(Q_{\text {trapped }}\right)$.

\begin{tabular}{lcccccc}
\hline \hline & \multicolumn{3}{c}{$\Delta N_{e}<0$} & \multicolumn{3}{c}{$\Delta N_{e}>0$} \\
& $E$ & $\sigma E$ & $Q_{\text {trapped }}$ & $E$ & $\sigma E$ & $Q_{\text {trapped }}$ \\
\hline$\Delta \vec{E}$ & - & - & - & + & + & + \\
$\Delta \lambda_{\text {deph }}$ & + & + & + & - & + & - \\
Total & +- & +- & +- & +- & ++ & +- \\
\hline \hline
\end{tabular}

expected effects are summarized in Table IV. Since the relative effects of the two processes are not known in advance, it is hard to make predictions on the net effect of density variations. The only exception seems to be the energy spread for higher plasma densities, which is expected to increase both due to the shorter dephasing length and the stronger wakefields.

The simulated effects of the density variations are shown in Fig. 6.

As can be seen in Fig. 6, the variations in the plasma density have almost no influence on the final energy or the trapped electron fraction. The average energy decreases slightly at both higher and lower densities, but the effect of changed dephasing length and accelerating fields compensate each other quite well. The energy spread does indeed strongly increase for increased density as predicted. For lower densities, the lower trapping fields lead to trapping over a smaller portion of the plasma wave which apparently decreases the effect of the changed dephasing length on the energy spread. This leads to a lower energy spread for lower densities. The amount of trapped electrons is mainly determined by the amplitude of the plasma wave: increasing with a higher amplitude. For lower densities, the lower trapping fields are apparently partially compensated by the longer dephasing length which allows the electrons

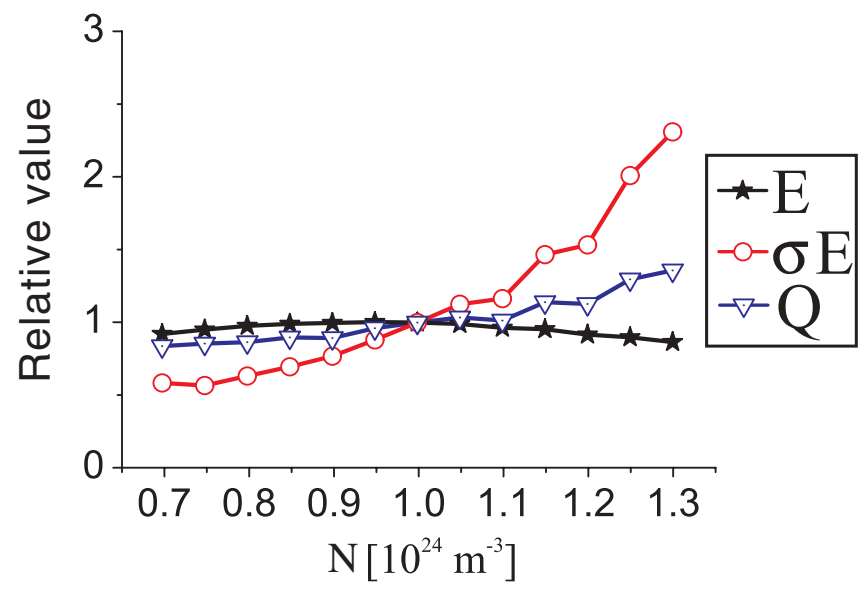

FIG. 6. (Color) Effect of fluctuations in the plasma density on the final beam parameters [energy $(E)$, energy spread $(\sigma E)$, and trapped fraction $(Q)]$ for a laser of $a_{0}=0.32$ (c) with injection $300 \mathrm{fs}$ behind the laser pulse. to be accelerated over a longer length before entering the defocusing phase of the plasma wave.

\section{Injection in front of the laser pulse}

A second approach to injecting electrons was proposed by Khachatryan $[9,30,31]$ where the electrons are injected in front of the laser pulse. This scheme generally requires a higher laser power than used for injection behind the laser. However, it has the advantage of compressing the initial bunch into a single final bunch, even if the initial bunch is several plasma wavelengths long. Since this approach behaves differently for various laser intensities, we will evaluate the effect of experimental variations in three different regimes of laser power: $10 \mathrm{TW}, 30 \mathrm{TW}$, and $100 \mathrm{TW}$. This corresponds to an $a_{0}$ of $0.32,0.56$, and 1.02 , respectively.

The electrons enter the plasma $300 \mathrm{fs}$ in front of the laser pulse in all three cases. This time has been chosen in order to reduce injecting part of the bunch after the laser pulse when considering timing variations of up to $500 \mathrm{fs}$ (Sec. IV C 2). Injecting the electrons further in front of the laser pulse will increase the time the laser needs to catch up to the electrons (Sec. IV C 1). Since both the laser pulse and electrons are traveling through the plasma with almost the speed of light in vacuum, this quickly leads to much longer required plasma lengths.

Again we first optimize the injection energy for maximum final energy, maximum trapped charge, and minimum energy spread and fix the plasma length at the point were these values are attained. The results of these optimizations are given in Table V. The injection energies found are in good agreement with those found in [9] with the correction for the larger initial electron bunch radius.

The resulting energy spectra of the accelerated electrons are shown in Fig. 7. When evaluating the effect of fluctuations in the various parameters we will again look at the relative changes of the three main characteristics of the accelerated bunches: energy, energy spread, and the number of trapped electrons relative to the number of injected electrons. As a reference, the values of these characteristics for single bunches at the optimized setting (Table V) are given in Table VI.

\section{Bunch evolution for injection before the laser pulse}

As a reference frame when looking at the effect of the different parameter fluctuations on the final beam proper-

TABLE V. Optimized electron and plasma parameters for the various laser regimes when injecting electron bunches $300 \mathrm{fs}$ in front of the laser pulse.

\begin{tabular}{lccc}
\hline \hline Laser vector potential $\left(a_{0}\right)[]$ & 0.32 & 0.56 & 1.02 \\
\hline Laser power @ 50 fs [TW] & 10 & 30 & 100 \\
Plasma length [mm] & 112.0 & 60.0 & 46.0 \\
Electron kinetic energy [MeV] & 8.15 & 4.60 & 1.79 \\
\hline \hline
\end{tabular}



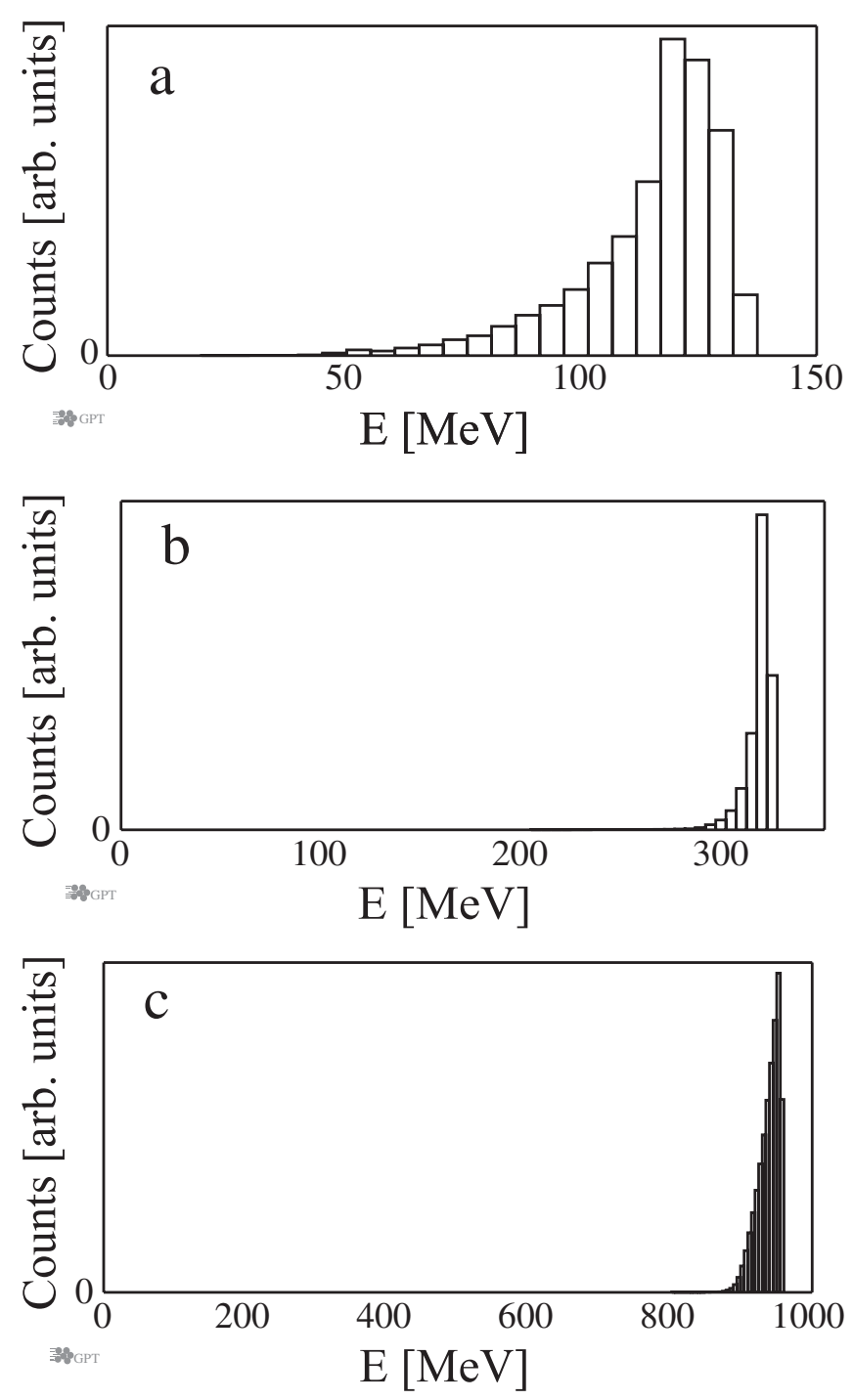

FIG. 7. Energy spectra of the accelerated bunches at their design settings (Table $\mathrm{V}$ ) for the different laser regimes: $a_{0}=$ 0.32 (a), $a_{0}=0.56$ (b), and $a_{0}=1.02$ (c) with injection $300 \mathrm{fs}$ in front of the laser pulse.

ties and to illustrate the differences in approach with injection after the laser pulse (Sec. IV B 1), we will first look at the normal bunch evolution of an electron bunch injected in front of the laser pulse (see $[9,30]$ for a more mathematical description). The typical evolution for an optimized electron bunch injected before the laser pulse is shown in Fig. 8.

At $t=0$, we again see the bunch before entering the plasma with all particles having the same energy and a Gaussian distribution in both time and radial direction. At $t=40 \mathrm{ps}$, the laser pulse is starting to catch up with the electrons which have been injected into the plasma $300 \mathrm{fs}$ before the laser: First, the electrons are slightly decelerated and then accelerated as they travel further back in the faster moving plasma wave (see also Fig. 1). At $t=86 \mathrm{ps}$, the
TABLE VI. Parameters of accelerated electron bunches in the various laser regimes when injecting $300 \mathrm{fs}$ in front of the laser pulse.

\begin{tabular}{lccc}
\hline \hline Laser vector potential $\left(a_{0}\right)[]$ & 0.32 & 0.56 & 1.02 \\
\hline Average electron energy $[\mathrm{MeV}]$ & 117 & 318 & 940 \\
Electron energy spread $[\mathrm{MeV}]$ & 12 & 4 & 13 \\
Electron fraction trapped [\%] & 82 & 89 & 95 \\
\hline \hline
\end{tabular}

laser pulse has almost completely moved past the electron bunch and the majority of the electrons is being accelerated. A small fraction is being expelled in the radial direction because they have moved into the radially defocusing phase of the plasma wave. However, most of the electrons stay in the phase of the plasma wave that is both accelerating and focusing. At $t=118 \mathrm{ps}$, almost all the electrons that did not gain enough energy to stay out of the defocusing phase of the plasma wave have been radially expelled and removed from the simulation. Also, some of the electrons are leaving the accelerating phase of the plasma wave and are no longer gaining energy, but are still in the focusing phase. At $t=204 \mathrm{ps}$, the electron bunch has just exited the plasma channel. At the exit, the bulk of the electrons were between the accelerating and decelerating phase of the plasma in the region of low longitudinal fields. The electrons in the front of the bunch were experiencing slightly decelerating fields while those in the back were still experiencing accelerating fields.

\section{Effects of timing variations}

As with injection behind the laser pulse (see Sec. IV B 2), we will first look at the effect of fluctuations in the timing between the laser pulse and the electrons. The maximum fluctuation in timing or jitter is again set at $500 \mathrm{fs}$ from the design point (which is $300 \mathrm{fs}$ in front of the laser pulse).

In general we would expect the following trends: Timing jitter will cause the electron beam to either not reach the dephasing phase (due to being injected too late) or to overshoot the dephasing phase and to enter the decelerating phase of the plasma wave. This leads to a decrease in average energy, an increase in energy spread while retaining the same trapped charge. The simulated effects of the timing variation on electrons injected $300 \mathrm{fs}$ in front of the laser pulse can be seen in Fig. 9.

For a wave plasma wave of $a_{0}=1.02$ [Fig. 9(c)], we see the expected trends except for timing variations of $\delta t<$ $-150 \mathrm{fs}$. At these timings, part of the electron bunch is injected after the laser pulse, which leads to a much larger energy spread, a lower average energy, and lower trapped charge. The radial profile of the beam at $\delta t=-300$ fs just after entering the plasma can be seen in Fig. 10. By comparing the radial profile with those from optimized injection before (Fig. 8) and behind (Fig. 3) the laser pulse, it can be seen that both processes are taking place at the 

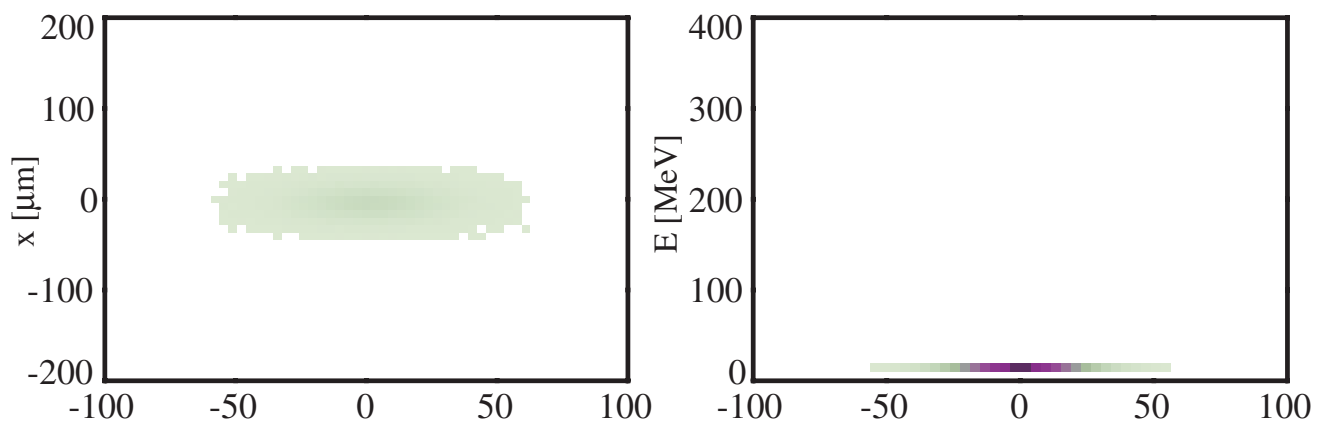

$\mathrm{t}=0 \mathrm{ps}$

$\mathrm{E}=4.6 \mathrm{MeV}$

$\sigma \mathrm{E}=0 \mathrm{MeV}$

$\mathrm{Q}=100 \%$
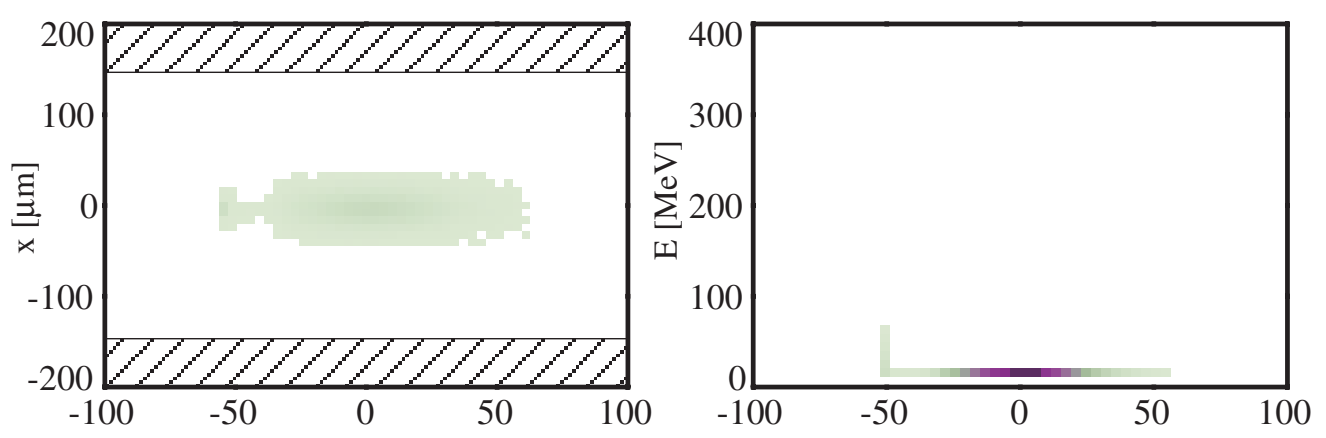

\begin{tabular}{|l}
$\mathrm{t}=40 \mathrm{ps}$ \\
$\mathrm{E}=4.9 \mathrm{MeV}$ \\
$\sigma \mathrm{E}=2.7 \mathrm{MeV}$ \\
$\mathrm{Q}=100 \%$
\end{tabular}
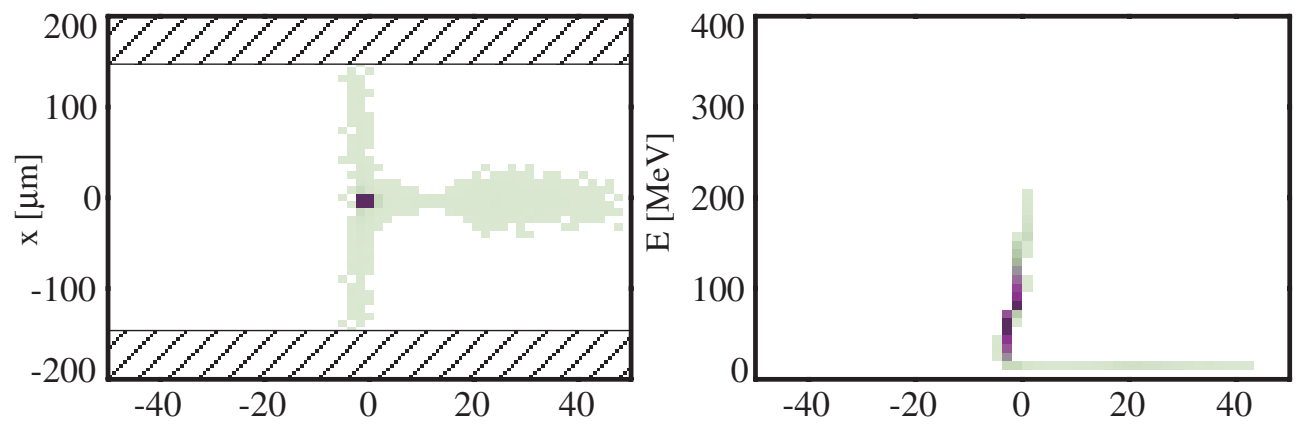

$\mathrm{t}=86 \mathrm{ps}$

$\mathrm{E}=67 \mathrm{MeV}$

$\sigma \mathrm{E}=42 \mathrm{MeV}$

$\mathrm{Q}=98 \%$
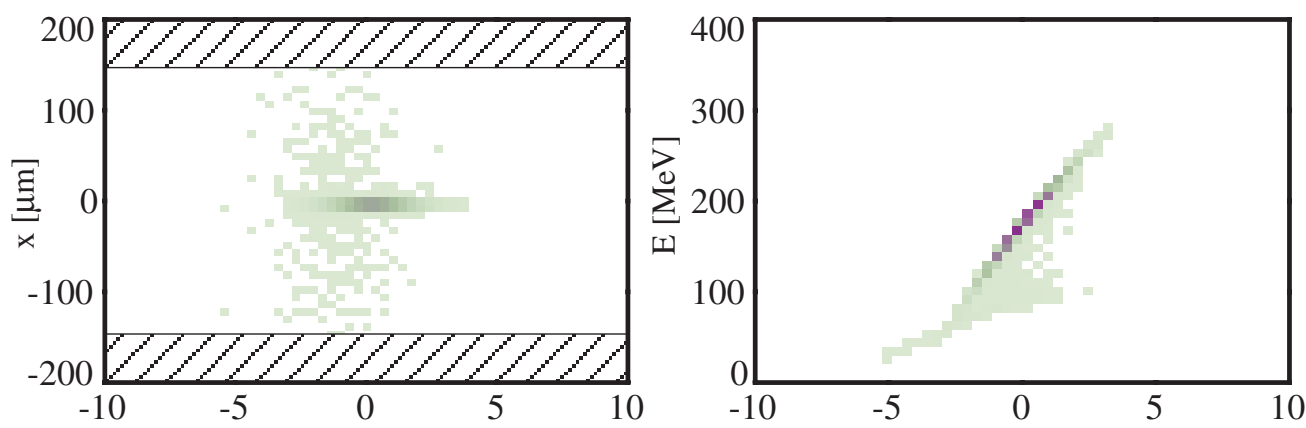

$\mathrm{t}=118 \mathrm{ps}$

$\mathrm{E}=169 \mathrm{MeV}$

$\sigma \mathrm{E}=45 \mathrm{MeV}$

$\mathrm{Q}=91 \%$
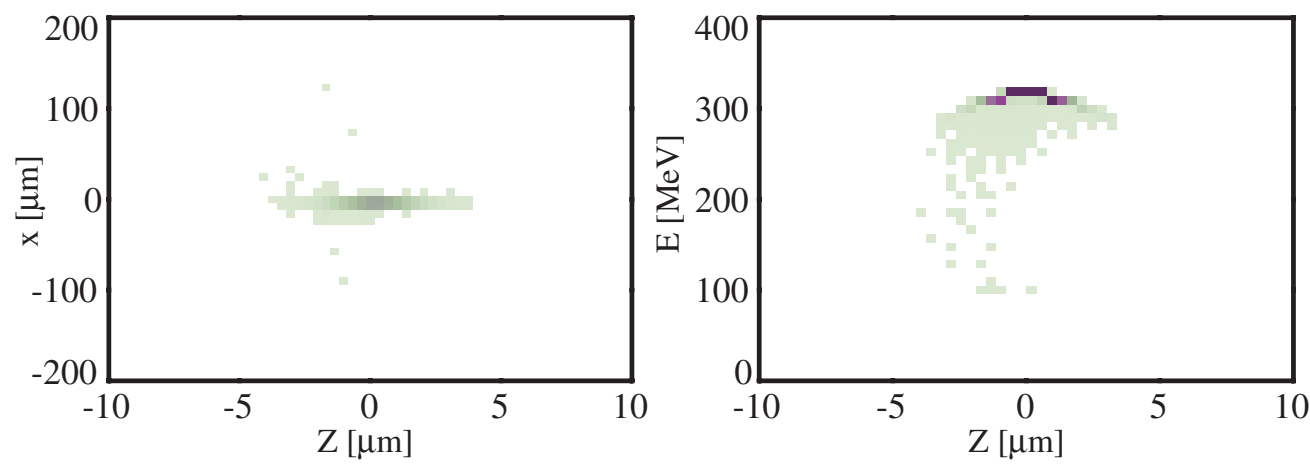

$\mathrm{t}=204 \mathrm{ps}$
$\mathrm{E}=317 \mathrm{MeV}$
$\sigma \mathrm{E}=11 \mathrm{MeV}$
$\mathrm{Q}=89 \%$

FIG. 8. (Color) Density plot of the time evolution for the radial profile (left) and energy (right) of an electron bunch injected $300 \mathrm{fs}$ in front of a plasma wave from a laser pulse with $a_{0}=0.56$. For each snapshot, the time $(t)$, energy $(E)$, energy spread $(\sigma E)$, and charge $(Q)$ are given on the right. 

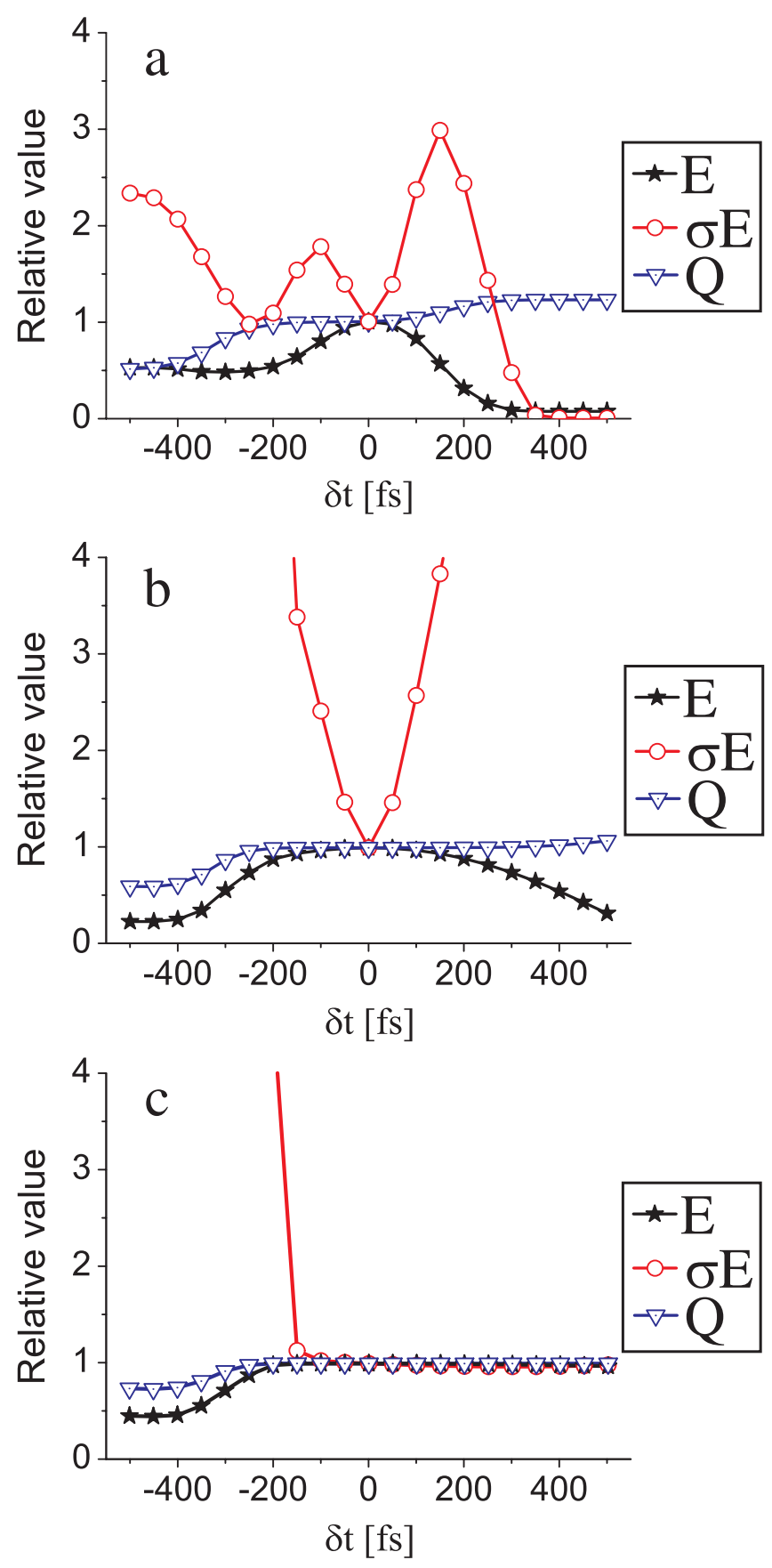

FIG. 9. (Color) Effect of $\pm 500 \mathrm{fs}$ fluctuations in the timing between laser and electrons $(\delta t>0$ means the electrons are injected further in front of the laser pulse) on the final beam parameters [energy $(E)$, energy spread $(\sigma E)$ and trapped fraction $(Q)]$ for the different injection regimes: $a_{0}=0.32(\mathrm{a}), a_{0}=0.56$ (b), and $a_{0}=1.02$ (c) with injection $300 \mathrm{fs}$ in front of the laser pulse.

same time while the electron injection energy and plasma length have only been optimized for injection before the laser pulse. The fact that the electron energy and plasma length have not been optimized for injection behind the laser pulse leads to a lower final energy and higher energy

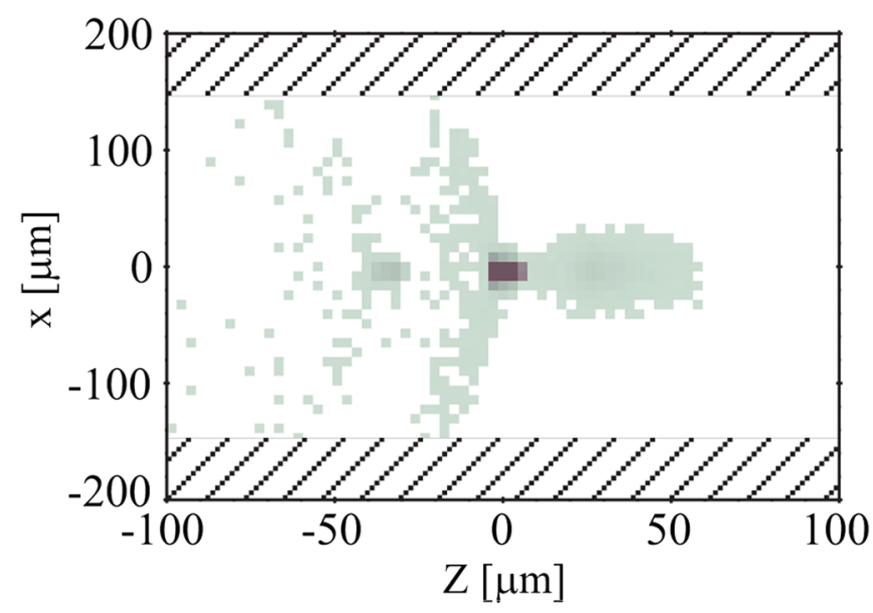

FIG. 10. (Color) Density plot of the radial profile for the electron beam just after entering the plasma with a plasma wave generated by a laser of $a_{0}=1.02$ and a timing variation of $\delta t=$ $-300 \mathrm{fs}$.

spread. Also, part of the electrons injected behind the laser pulse will be in the defocusing phase of the plasma wave and will be radially ejected, leading to a lower trapped charge. The part of the electron bunch that does get injected before the laser pulse will also interact with the plasma wave over a longer distance compared to those injected at the nominal time, leading to dephasing which causes an additional lowering of the final energy and increase of the energy spread.

When comparing the effects on timing variations on injection of electrons in front of plasma waves of $a_{0}=$ 0.32 and $a_{0}=0.56$ [Fig. 9(a) and 9(b)], we see a similar behavior. In these regimes, the sensitivity to timing variations seems much greater when compared to the high intensity regime [Fig. 9(c)] or injection after the laser pulse (Fig. 4). To explain the relevant effects, we will look closer at the situation of Fig. 9(a) $\left(a_{0}=0.32\right)$.

When the electron bunch is injected far in front of the laser pulse $(\delta t>400$ fs or more than $700 \mathrm{fs}$ in front of the laser), the electrons exit the plasma before the laser pulse has had a chance to catch up with them, they retain their original energy (spread) and no charge is radially expelled. For electrons injected slightly early ( 0 fs $<\delta t<400 \mathrm{fs}$ ), the total interaction time between the plasma wave and the electrons is shorter and the electrons have not reached the dephasing phase yet leading to a lower energy and large energy spread. Also, not all electrons that are radially expelled have reached the wall yet, leading to a higher (apparent) charge.

Electrons injected slightly too late $(-150$ fs $<\delta t<$ $0 \mathrm{fs}$ ) will spend a longer time in the plasma wave, which means they are already entering the decelerating phase of the plasma wave before the plasma ends. This again leads to a lower energy and higher energy spread. As soon as part of the electron bunch is injected behind the laser pulse $(-500 \mathrm{fs}<\delta t<-150 \mathrm{fs}$ ), we see a decrease in the 
trapped charge as those electrons injected in the defocusing phase of the plasma wave are radially expelled. Since about half of the total phase of the plasma wave is defocusing, the trapped fraction also drops to about half of the electrons. There is also an increase in energy spread and lowering of the average energy as the electrons injected both before and behind the laser pulse reach the decelerating phase of the plasma wave and are slowed down. Part of the electrons injected behind the laser pulse are even slowed down enough to move back to the accelerating phase (since they are now no longer moving faster than the plasma wave) and get reaccelerated.

In the case with a laser with $a_{o}=0.56$ [Fig. 9(b)], we see the same effects as in the case of a laser with $a_{o}=0.32$ [Fig. 9(a)] discussed above. Since the electrons in this case have a lower injection energy, the laser pulse catches up to them faster, thus the timing variation needed for the electron bunch to keep ahead of the laser pulse is larger. Also, the energy spread can be more than 20 times the nominal value [outside the scale of Fig. 9(b)]. This larger increase in energy spread compared to the case of $a_{o}=0.32$ [Fig. 9(a)] is due to two reasons: First, the relative energy spread at the nominal settings is lower (see Table VI) which means the same energy spread leads to a larger value relative to the nominal value. Second, the maximum energy in this case is larger which means the difference between electrons that are accelerated to the maximum energy and those that have been decelerated is larger as well.

\section{Effects of laser energy variations}

In the wave-breaking regime [2-6] where electrons are injected from the plasma itself in the same regime of laser power as considered here (10-100 TW), fluctuations in the laser pulse energy are one of the major contributions to the lower reproducibility of the electron bunches produced. The laser power has to be accurately tuned to just above or just below wave breaking in order to produce beams with desired properties. We will again consider fluctuations up to $10 \%$ from the nominal energy and look at the effects of these fluctuations on externally injected electron bunches. As mentioned before (Sec. IV B 3), a laser pulse with a lower energy/intensity will lead to a plasma wave with lower amplitude. This is again expected to lead to lower final energy, lower trapped charge, and lower energy spread (since a smaller part of the plasma wave is capable of trapping the electrons); the reverse would hold for laser pulses with a higher intensity. The simulation results for laser power variations in the different power regimes is shown in Fig. 11.

In Figs. 11(b) and 11(c) we see the behavior generally expected. From linear theory $[8,28,32-34]$ it follows that the wave amplitude scales with the square root of the laser pulse energy. This means a $10 \%$ variation in pulse energy should result in a 5\% variation in final energy. This is the
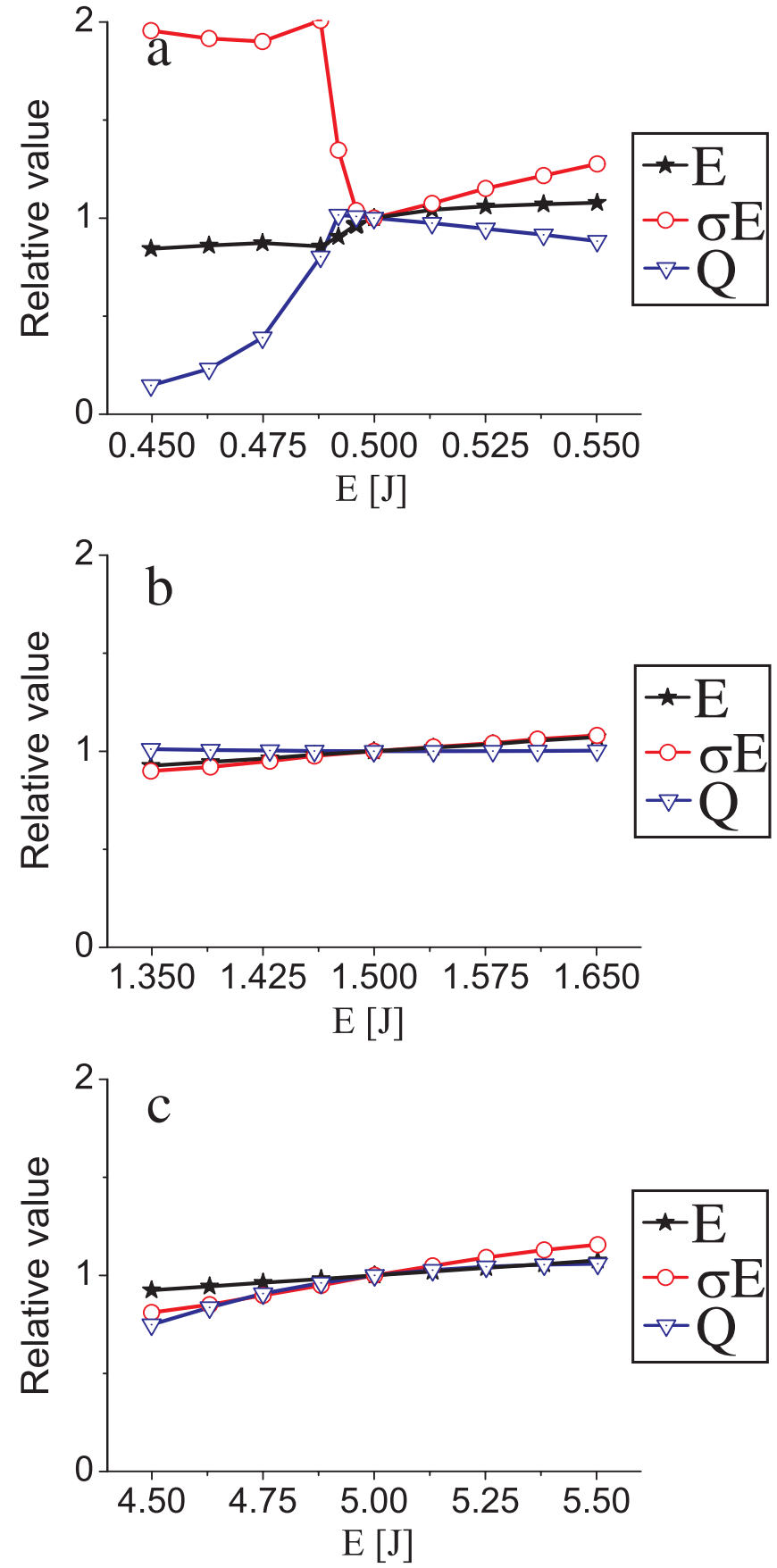

FIG. 11. (Color) Effect of $\pm 10 \%$ fluctuations in the laser energy on the final beam parameters [energy $(E)$, energy spread $(\sigma E)$, and trapped fraction $(Q)$ ] for the different injection regimes: $a_{0}=0.32$ (a), $a_{0}=0.56$ (b), and $a_{0}=1.02$ (c) with injection $300 \mathrm{fs}$ in front of the laser pulse.

case for the laser pulse with $a_{0}=0.56$, but for the more nonlinear case of $a_{0}=1.02$, we see a slightly larger variation of the final energy of $7.5 \%$. In the case of a plasma wave of $a_{0}=1.02$ [Fig. 11(c)], it is seen that lower intensity of the laser pulses also results in a decrease of the amount of trapped charge. Even for a laser energy variation 
of $-10 \%$, the trapped charge for a laser pulse with a nominal $a_{0}$ of 0.56 remains the same. In the case of a nominal $a_{0}$ of 1.02 , the same $-10 \%$ variation of laser pulse energy results in a clear decrease in trapped charge as the accelerating fields are no longer strong enough to trap the same number of electrons trapped in the nominal case.

In Fig. 11(a), we see a different kind of behavior, mainly due to the fact that the strength of the plasma wave is very close to the limit needed to trap electrons. At lower laser power, a large part of the electron bunch, those further from the optical axis, is no longer trapped and focused to the optical axis. This is due to the radially decreasing strength of the plasma wave. These particles are either radially expelled to the channel wall or will travel at a large radius on the outside the plasma wave. The former leads to a lower trapped fraction while the latter results in a higher energy spread and lower average energy. The effect of this process is illustrated in Fig. 12.

From the radial distribution in Fig. 12, it can be seen that fluctuations in the laser pulse energy can have significant effects on the amount of radial focusing of the final bunches. If the laser pulse energy is below the design setting $(E=0.45 \mathrm{~J}, E=0.4875 \mathrm{~J})$, there is a significant halo of low energy electrons around the main bunch leading to the increased energy spread. At the design setting $(E=0.50 \mathrm{~J})$, the radial fields are strong enough to expel most of these electrons far enough from the plasma wave to be removed. For higher laser pulse energy $E=0.5175 \mathrm{~J}$, $E=0.55 \mathrm{~J}$ ), the plasma wave is strong enough to trap electrons at a larger radius, but these electrons experience a lower accelerating field than those at the optical axis, thus resulting in an increased energy spread.

\section{Effects of density variations}

For the case of injection before the laser pulse, the expected results for variations of the plasma density will be the same as for the case of injection behind the laser pulse (see Table IV). This means that it is again hard to predict from simple principles what the effects of density variations will be on the final bunch parameters. The
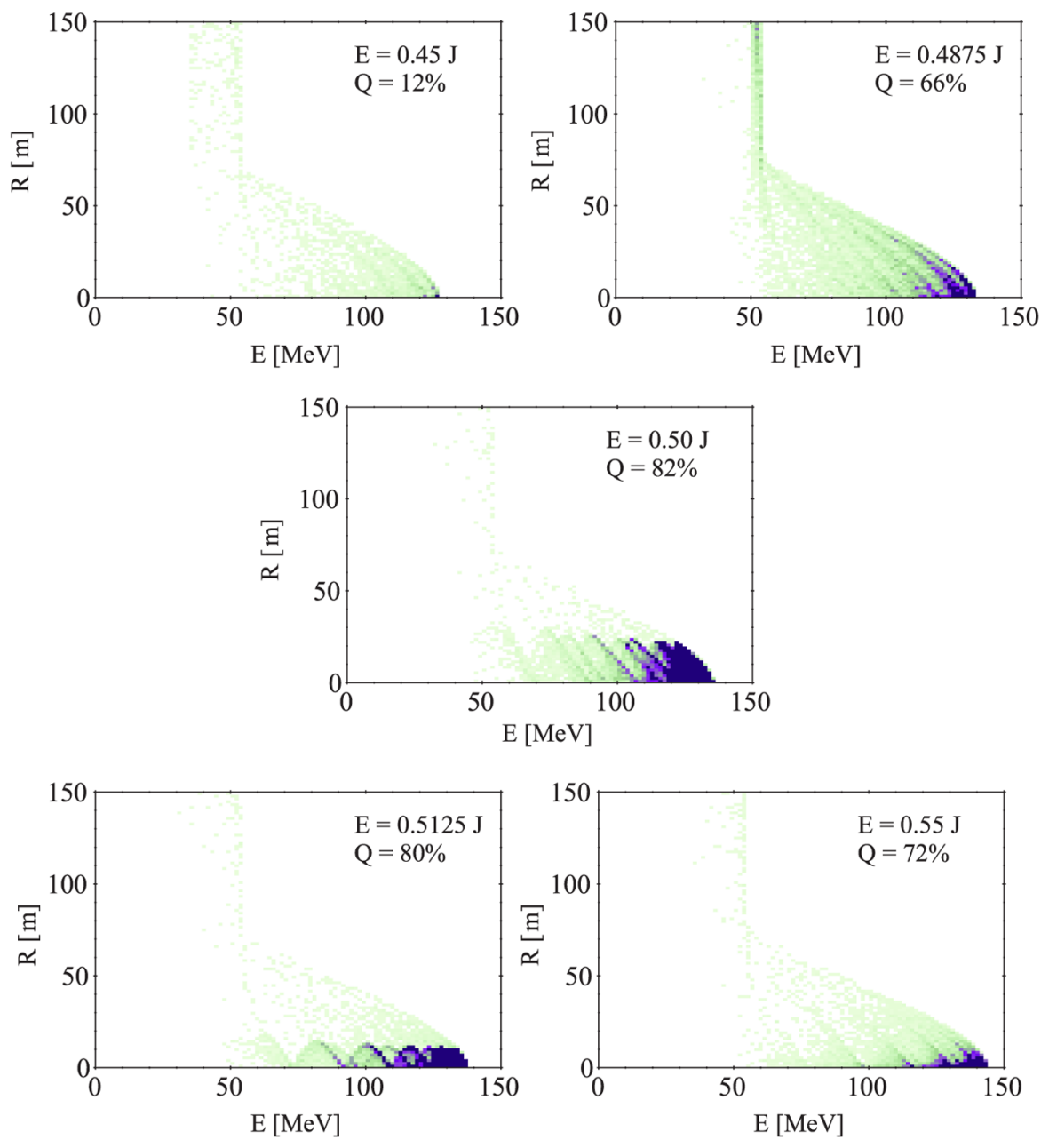

FIG. 12. (Color) Density plot of the radial distribution vs energy for electron bunches injected 300 fs in front of the laser pulse in the bunch after exiting the plasma channel with a plasma wave of $a_{0}=0.32$ for various laser energy variations given in Fig. 11 . 
simulated effects of the density variations for electrons injected in front of the laser pulse can be seen in Fig. 13.

In all three laser power regimes, the effect of density fluctuations on the final energy is dominated by the changing dephasing length. For lower than nominal densities, the longer time spent in the accelerating phase more than
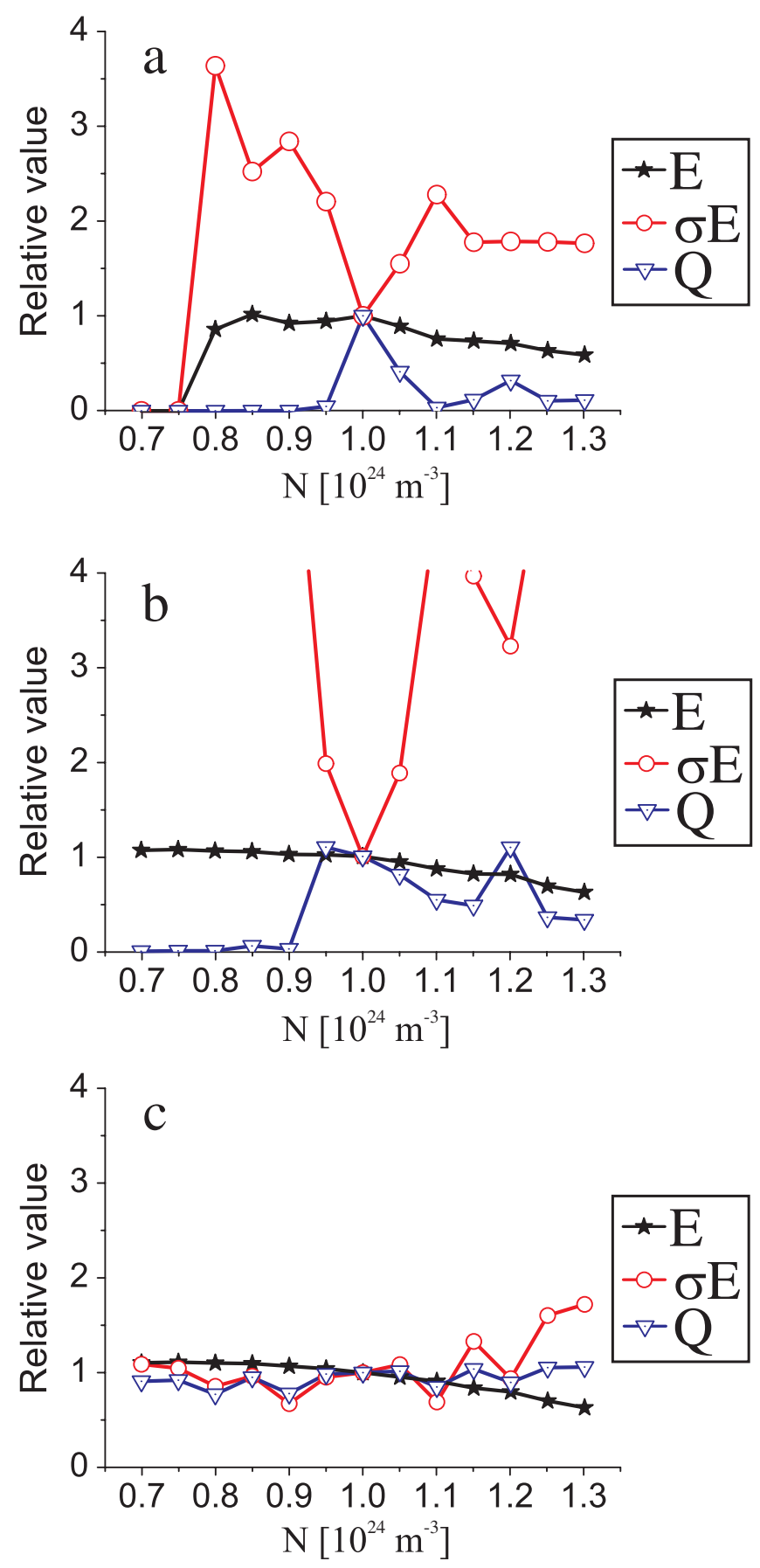

FIG. 13. (Color) Effect of $\pm 30 \%$ fluctuations in the plasma density on the final beam parameters [energy $(E)$, energy spread $(\sigma E)$, and trapped fraction $(Q)]$ for the different injection regimes: $a_{0}=0.32$ (a), $a_{0}=0.56$ (b), and $a_{0}=1.02$ (c) with injection $300 \mathrm{fs}$ in front of the laser pulse. compensates the lower accelerating fields. Since the length of the plasma is optimized for the nominal density however, the electrons do not reach their maximum attainable energy for these densities and laser powers. In the case of higher than nominal densities, the electrons have already entered the decelerating phase of the plasma wave before reaching the end of the plasma and will thus have a lower final energy, despite the higher fields in the plasma wave.

In the higher laser power regime $\left[a_{0}=1.02\right.$, Fig. 13(c)], the energy spread for densities below the nominal value is increased due to the fact that the electrons have not reached dephasing yet. For higher than nominal densities, the lower final energy due to the shorter dephasing length also limits the energy spread. For a density of $1.3 \times 10^{24} \mathrm{~m}^{-3}$, the energy spread is increased again because the increased radial fields cause electrons further from the optical axis to get trapped as well, leading to lower final energies. The behavior of the amount of trapped charge is determined by the change in the radial focusing fields and the change in trapping time: For lower than nominal densities, the trapping fields are lower, but due to the increased dephasing length, the time electrons can be accelerated before reaching the defocusing phase is increased. For higher than nominal densities, higher trapping fields can work over a shorter time span. The result of these two opposite effects can be seen in Fig. 13(c).

In Figs. 13(a) and 13(b), we see a very strong sensitivity to fluctuations of the plasma density. The energy spread for densities below the nominal value is increased due to the electrons not having reached the dephasing point yet. However, for densities below $0.9 \times 10^{24} \mathrm{~m}^{-3}$, the fields of the plasma wave are no longer strong enough to trap more than a few electrons from the injected bunch. This also means that the effect on the average energy of the outlier electrons that have not been radially ejected far enough can have a significant impact on the energy spread as well as the final energy. In the case of a laser intensity of $a_{0}=0.32$ and a density below $0.8 \times 10^{24} \mathrm{~m}^{-3}$, all the electrons are radially ejected resulting in no final trapped electrons.

For higher than nominal densities, there is an oscillatory behavior of both the trapped charge and the energy spread as a function of the density: First the trapped charge decreases at $1.1 \times 10^{24} \mathrm{~m}^{-3}$ and the energy spread increases, then the trapped charge increases again until $1.2 \times$ $10^{24} \mathrm{~m}^{-3}$ while the energy spread decreases. From $1.2 \times$ $10^{24} \mathrm{~m}^{-3}$ to $1.3 \times 10^{24} \mathrm{~m}^{-3}$, the trapped charge decreases again. This same behavior is visible for both $a_{0}=0.32$ and $a_{0}=0.56$. The reason for this strange behavior becomes more clear when we look at the particle trajectories for identical particles from a bunch for different plasma densities as is shown in Fig. 14. Here it is seen that the radial betatron oscillations due to the radial electric fields and the azimuthal magnetic field of the plasma wave are much 

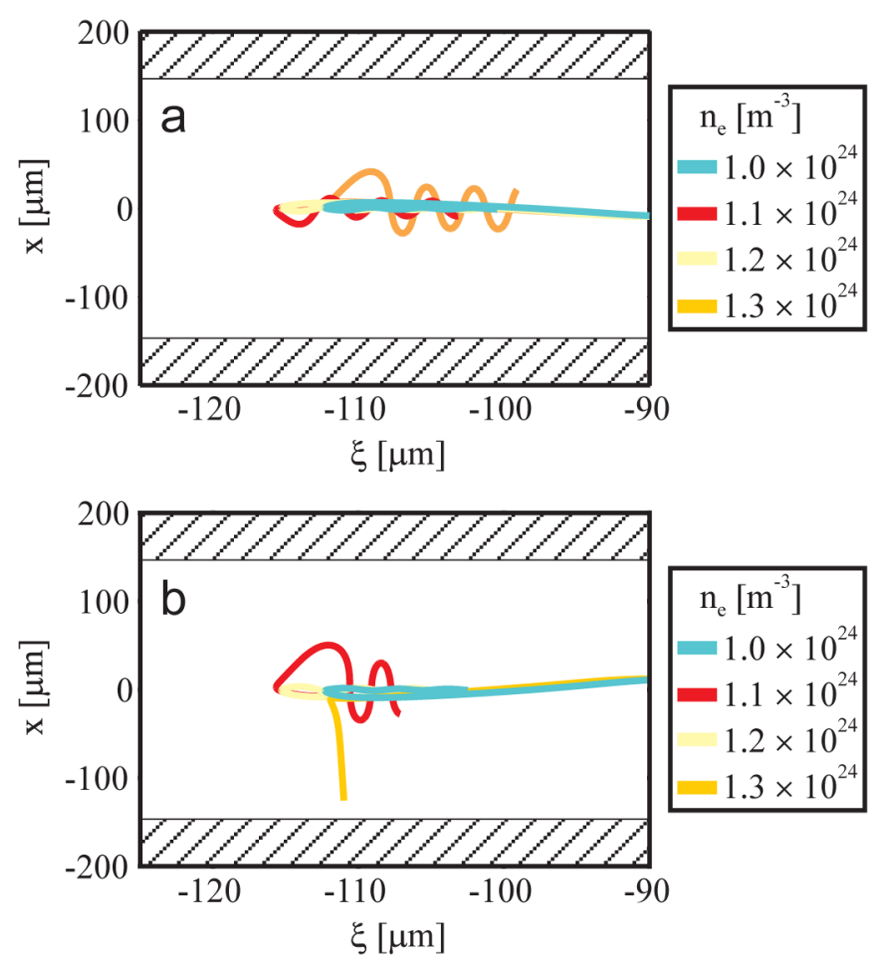

FIG. 14. (Color) Particle trajectories in the comoving coordinate $\xi$ [see Eq. (7)] for two different test particles for densities of $1.0-1.3 \times 10^{24} \mathrm{~m}^{-3}$ illustrating the difference in betatron oscillations. Fluctuations in density can lead to both increased betatron oscillations (a) and can even lead to additional loss of electrons as the betatron oscillations expel them beyond the radius where they are still trapped (b).

larger for densities of $1.1 \times 10^{24} \mathrm{~m}^{-3}$ and $1.3 \times 10^{24} \mathrm{~m}^{-3}$ while they seem suppressed for a density of $1.2 \times$ $10^{24} \mathrm{~m}^{-3}$. These oscillations enhance the radial expulsion of electrons since the electrons are on average further away from the optical axis where the accelerating fields are the largest (see Fig. 1). This results in a lower energy and in the electrons slipping into the defocusing phase of the plasma wave.

\section{CONCLUSIONS}

We have described a method of combining a relativistic fluid model for the plasma wave with particle tracking for the electron bunch to simulate laser wakefield acceleration of externally injected electrons. Using this model, we investigated the effects of fluctuations in several key parameters as they may occur in practice during the acceleration of electrons from an externally injected electron bunch both behind and in front of a laser pulse generating a plasma wave: laser-electron synchronization, laser pulse energy, and plasma density. These effects were studied in three different regimes ranging from linear to nonlinear plasma waves.

Predicting the effects of the variations using qualitative theoretical trends can become especially difficult when, e.g., in the case of a variation in the plasma density, several effects are interacting simultaneously. The combined effects of dephasing, plasma wave amplitude changes, and (enhanced) betatron oscillations are hard, if not impossible to predict from qualitative trends since they can both enhance and frustrate each others separate effects.

Injection after the laser pulse gives lower final energy and trapped fraction but a smaller energy spread and is much more robust against variations of the different parameters for low laser intensities. This is especially true with regards to the timing stability, where injection in front of the laser pulse, even with the best synchronization thus far reported ( $\sim 100 \mathrm{fs}$, see Ref. [27]), can still lead to more than a doubling of the energy spread and a $20 \%$ decrease in average energy. For all the variations studied, the final energy of the electrons varies only slightly. The energy spread generally increases with fluctuations unless the amount of electrons trapped is also reduced. The amount of trapped electrons increases with stronger wave fields (higher density or more energy per laser pulse) and when injecting later into the plasma wave. For densities and laser pulse energy that are lower than the nominal settings, there is a decrease in trapped charge as the plasma wave can no longer trap all the electrons it trapped at the nominal settings.

For injection in front of the laser pulse, we see that injection in the high intensity regime $\left(a_{0}=1.02\right)$ is less influenced by variations in synchronization and plasma density than those of more linear plasma waves. If synchronization jitter causes the injection of part of the electron bunch behind the laser pulse however, a sharp increase of the energy spread is the result.

The electrons accelerated by a laser pulse with $a_{0}=$ 0.32 are very sensitive to fluctuations in any of the three parameters studied and even small variations can lead to a rapid increase of the energy spread and decrease of the amount of trapped electrons.

For the case of a laser pulse with $a_{0}=0.56$, we see only small effects due to variations in the laser pulse power, but both variations in the density and the synchronization cause a large increase in the energy spread of more than 20 times the optimized value.

Regarding the injection energy of the electrons, it also becomes clear that choosing the electron energy just above the trapping limit of the plasma wave, which leads to a low energy spread at the design specs, can lead to a great sensitivity to changes of that trapping limit due to variations in the plasma density or laser energy.

Overall, it appears that in the lower intensity regime, injection behind the laser pulse is the least sensitive to fluctuations in synchronization, laser pulse energy, and density. Injection of electrons in front of the laser pulse becomes less sensitive to fluctuations at intensities above $a_{0}=1$. Injecting in front of the laser at intensities below $a_{0}=1$ can yield electron bunches with high energy, low 
energy spread, and high trapping efficiency, but even small fluctuations in experimental parameters can cause the energy spread to increase manifold and the amount of trapped electrons to decrease.

\section{A. Multibunch characteristics}

For most practical purposes, it is not a single shot-toshot variation that matters, but the combined effects of fluctuations on a multishot experiment or application. We will therefore lastly consider the effect of multiple fluctuations. The fluctuations are assumed to follow a Gaussian distribution during a multishot experiment. As a starting point, we will take the case of injection of a electron bunch $300 \mathrm{fs}$ in front of a laser pulse with $a_{0}=1.02$ since, on average, this case seems to be the least sensitive to variations in timing, laser pulse energy, and plasma density. In Fig. 15 we see the energy spectra of the same multishot experiment assuming no fluctuations [Fig. 15(a)], a timing jitter with a standard deviation of $150 \mathrm{fs}$ [Fig. 15(b)], a variation in the laser pulse energy with a standard deviation of 5\% [Fig. 15(c)], and a variation in the plasma density with a standard deviation of 5\% [Fig. 15(d)] and 10\% [Fig. 15(e)].

As can be seen from the energy spectra in Fig. 15, a timing jitter of $150 \mathrm{fs}$ [Fig. 15(b)] still produces an energy spectrum quite similar to the one at the design settings [Fig. 15(a)], but a variation in the laser pulse energy of 5\% [Fig. 15(c)] or the same 5\% variation in the plasma density [Fig. 15(d)] leads to an energy spectrum that is at least twice as broad. A density variation of $10 \%$ [Fig. 15(e)] leads to an energy spectrum with broad features and a multibunch energy spread that is over 4 times that predicted by single bunch simulations at the design settings. It is therefore necessary to improve the measurement and control over the plasma density in order to reduce the density variations from the reported level of $20 \%$ down to $<5 \%$ in order to maintain the low energy spread of the single bunch experiments in a multibunch experiment.

\section{OUTLOOK}

The method presented in this paper can be used to predict the effects of unavoidable fluctuation in the parameters of current experiments on externally injected laser wakefield acceleration. The method can also be used to determine the maximum allowed variations of the specific parameters of these experiments in order to ensure a desired reproducibility. Moreover, it allows optimization of the design in order to minimize the effects of variations. The method allows these studies to be performed in a modest amount of time and enables the incorporation of the entire injection beam line. Furthermore, the method allows the inclusion of laser wakefield acceleration as a "module" into conventional accelerator design/codes.

Finally, the separate plasma wave module allows the implementation of other, more advanced, plasma wave
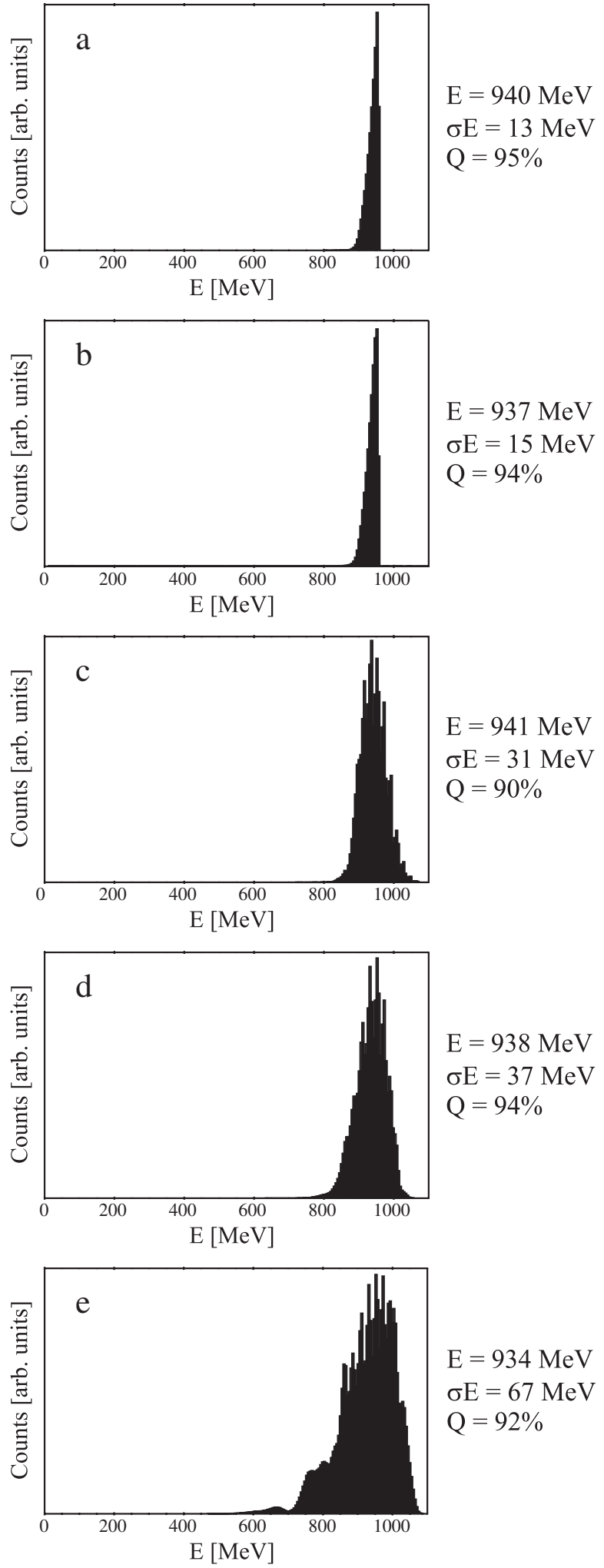

FIG. 15. Energy spectra of the accelerated bunch for a multishot experiment for $a_{0}=1.02$ with injection $300 \mathrm{fs}$ in front of the laser pulse under the following conditions: (a) design settings (Table V), (b) timing variation with $\sigma_{t}=150 \mathrm{fs}$, (c) Laser pulse energy with $\sigma_{\text {laser }}=5 \%$, (d) plasma density variations with $\sigma_{n_{e}}=5 \%$, and (e) plasma density variations with $\sigma_{n_{e}}=10 \%$. 
models to take into account effects such as laser depletion and laser pulse evolution while keeping the rest of the method intact.

\section{ACKNOWLEDGMENTS}

This work is part of the research program of the "Stichting voor Fundamenteel Onderzoek der Materie (FOM)." which is financially supported by the "Nederlandse Organisatie voor Wetenschappelijk Onderzoek (NWO)." We acknowledge the support of the European Community-New and Emerging Science and Technology Activity under the FP6 Structuring the European Research Area program (project EuroLEAP, Contract No. 028514).

\section{APPENDIX: CALCULATING THE EFFECTIVE BUNCH DENSITY}

As mentioned in Sec. II C, one of the assumptions that allows the decoupling of the evolutions of the plasma wave and the electron bunch is the fact that the effect of the electron bunch on the plasma can be neglected. This is the case when the effective density of the electron bunch is much smaller than the density of the plasma:

$$
n_{e_{\text {bunch }}}^{\text {eff }} \ll n_{e_{\text {plasma }}} \text {. }
$$

Since the radial electric fields of a relativistic electron bunch are Lorentz contracted, we must take this effect into account when looking at a worst case scenario:

$$
n_{e_{\text {bunch }}}^{\text {eff }} \leq n_{e_{\text {bunch }}} \gamma_{\text {bunch. }}
$$

With the data from the injected electron bunches (Tables I, II, and V), we can now make an estimate of the maximum effective bunch density:

$$
\begin{aligned}
n_{e_{\text {bunch }}}^{\text {eff }} & =\frac{Q}{\pi R^{2} L} \gamma_{\text {bunch }} \\
& =\frac{\left(\frac{10 \times 10^{-12}}{e}\right)}{\pi\left(30 \times 10^{-6}\right)^{2}\left(150 \times 10^{-15} c\right)} \times 17 \\
& =8.3 \times 10^{21} \ll 1.0 \times 10^{24} \mathrm{~m}^{-3} .
\end{aligned}
$$

So we can conclude that the effective density of the injected bunches is much smaller than the plasma density and thus the effect of beam loading can be neglected for the injected bunches.

However, the accelerated bunches can have much higher energy and a far shorter duration. For the case of a laser intensity of $a_{o}=1.02$, the effective density can become comparable or even greater than the plasma density. This means that for this regime beam loading effects might not be negligible.

For an estimate of the 3D beam loading limit $\left(N_{\mathrm{bl}}\right)$, we can use the estimate given in [9] [Eq. (19)]:
TABLE VII. Effect of space charge on the parameters of accelerated electron bunches when injecting $300 \mathrm{fs}$ in front of the laser pulse with $a_{0}=1.02$.

\begin{tabular}{lcc}
\hline \hline Parameter & $\begin{array}{c}\text { No space } \\
\text { charge }\end{array}$ & $\begin{array}{c}\text { Space } \\
\text { charge }\end{array}$ \\
\hline Average electron energy [MeV] & 940 & 931 \\
Electron energy spread [MeV] & 13 & 17 \\
Electron fraction trapped [\%] & 95 & 82 \\
\hline \hline
\end{tabular}

$$
N_{\mathrm{bl}}=\frac{\pi R^{2} n_{p} E_{z, \max }}{\left[1-R K_{1}(R)\right] k_{p}^{3}} .
$$

Here $R$ is the (normalized) bunch radius, $n_{p}$ the plasma density, $E_{z, \max }$ the (normalized) laser wake wave amplitude, $K_{1}(R)$ the modified Bessel function, and $k_{p}$ the plasma wave number. For the bunch in the case of a laser intensity of $a_{o}=1.02 \quad\left[R=0.21 \quad(7 \mu \mathrm{m}), \quad n_{p}=\right.$ $1.0 \times 10^{24} \mathrm{~m}^{-3}, E_{z, \max }=0.36\left(34.6 \frac{\mathrm{GV}}{\mathrm{m}}\right)$ ], Eq. (A4) gives a beam loading limit of $25 \mathrm{pC}$. This means that the charge chosen is still well below the beam loading limit.

Since the GPT code does allow for the inclusion of spacecharge effects, it is possible to study the effects of beam loading. The inclusion of space-charge effects does however significantly increase the computational power needed. The beam loading effects of the $10 \mathrm{pC}$ bunch charge for a laser intensity of $a_{o}=1.02$ can be found in Table VII.

As can be seen from Table VII, inclusion of beam loading effects only causes a small decrease of the final energy $(<1 \%)$. There is however an increase in the energy spread and a decrease in the trapped charge (more electrons get radially expelled) due to the repulsive forces within the electron bunch.

[1] T. Tajima and J. M. Dawson, Phys. Rev. Lett. 43, 267 (1979).

[2] S. P. D. Mangles et al., Nature (London) 431, 535 (2004).

[3] C. G. R. Geddes, C. Toth, J. van Tilborg, E. Esarey, C. B. Schroeder, D. Bruhwiler, C. Nieter, J. Cary, and W.P. Leemans, Nature (London) 431, 538 (2004).

[4] J. Faure, Y. Glinec, A. Pukhov, S. Kiselev, S. Gordienko, E. Lefebvre, J.-P. Rousseau, F. Burgy, and V. Malka, Nature (London) 431, 541 (2004).

[5] W. P. Leemans, B. Nagler, A. J. Gonsalves, C. Toth, K. Nakamura, C. G. R. Geddes, E. Esaray, C. B. Schroeder, and C. J. Hooker, Nature Phys. 2, 696 (2006).

[6] J. Faure, C. Rechatin, A. Norlin, A. Lifschitz, Y. Glinec, and V. Malka, Nature (London) 444, 737 (2006).

[7] A. Brantov, T. Esirkepov, M. Kando, H. Kotaki, V. Bychenkov, and S. Bulanov, Phys. Plasmas 15, 073111 (2008).

[8] D. F. Gordon, R. F. Hubbard, J. H. Cooley, B. Hafizi, A. Ting, and P. Sprangle, Phys. Rev. E 71, 026404 (2005). 
[9] A. G. Khachatryan, F. A. van Goor, K.-J. Boller, A. J. W. Reitsma, and D. A. Jaroszynski, Phys. Rev. ST Accel. Beams 7, 121301 (2004).

[10] A. F. Lifschitz, J. Faure, V. Malka, and P. Mora, Phys. Plasmas 12, 093104 (2005).

[11] W. H. Urbanus, W. van Dijk, S. B. van der Geer, G. J. H. Brussaard, and M. J. van der Wiel, J. Appl. Phys. 99, 114501 (2006).

[12] A. Pukhov, J. Plasma Phys. 61, 425 (1999).

[13] R. G. Hemker, F. S. Tsung, V. K. Decyk, W. B. Mori, S. Lee, and T. Katsouleas, Proceedings of the 1999 Particle Accelerator Conference, edited by A. Luccio and W. MacKay (IEEE, Piscataway, 1999), Vol. 5, p. 3672.

[14] C. Nieter and J. R. Cary, J. Comput. Phys. 196, 448 (2004).

[15] W. van Dijk, S. van der Geer, M. van der Wiel, and G. Brussaard, Phys. Plasmas 15, 093102 (2008).

[16] General Particle Tracer (GPT) code, Pulsar Physics, Eindhoven, http://www.pulsar.nl.

[17] P. Volfbeyn, E. Esarey, and W. P. Leemans, Phys. Plasmas 6, 2269 (1999).

[18] P. Sprangle, E. Esarey, A. Ting, and G. Joyce, in AIP Conference Proceedings: Advance Accelerator Concepts (AIP, New York, 1989), Vol. 193, pp. 376-387.

[19] F. Dorchies et al., Phys. Rev. Lett. 82, 4655 (1999).

[20] V. V. Ivanov, P. S. Antsiferov, K. N. Koshelev, M. R. Akdim, and F. Bijkerk, Phys. Rev. Lett. 97, 205007 (2006).

[21] N. Andreev, E. Chizhonkov, and L. Gorbunov, Russ. J. Numer. Anal. Math. Modelling 13, 1 (1998).

[22] P. Mora and T. A. Antonsen, Jr., Phys. Plasmas 4, 217 (1997).
[23] A. Butler, D. J. Spence, and S. M. Hooker, Phys. Rev. Lett. 89, 185003 (2002).

[24] N. A. Bobrova, A. A. Esaulov, J. I. Sakai, P. V. Sasorov, D. J. Spence, A. Butler, S. M. Hooker, and S. V. Bulanov, Phys. Rev. E 65, 016407 (2001).

[25] B. Broks, W. van Dijk, and J. J. A. M. van der Mullen, J. Phys. D 39, 2377 (2006).

[26] B. H. P. Broks, K. Garloff, and J. J. A. M. van der Mullen, Phys. Rev. E 71, 016401 (2005).

[27] F. B. Kiewiet, A.H. Kemper, O.J. Luiten, G. J. H. Brussaard, and M.J. van der Wiel, Nucl. Instrum. Methods Phys. Res., Sect. A 484, 619 (2002).

[28] H. Dewa et al., Nucl. Instrum. Methods Phys. Res., Sect. A 410, 357 (1998).

[29] A. J. Gonsalves, T.P. Rowlands-Rees, B. H. P. Broks, J. J. A. M. van der Mullen, and S. M. Hooker, Phys. Rev. Lett. 98, 025002 (2007).

[30] A. G. Khachatryan, Phys. Rev. E 65, 046504 (2002).

[31] A. Khachatryan, M. Luttikhof, A. Irman, F. van Goor, J. Verschuur, H. Bastiaens, and K.-J. Boller, Nucl. Instrum. Methods Phys. Res., Sect. A 566, 244 (2006).

[32] R. Hubbard, D. Gordon, J. Cooley, B. Hafizi, T. Jones, D. Kaganovich, P. Sprangle, A. Ting, A. Zigler, and J. Dexter, IEEE Trans. Plasma Sci. 33, 712 (2005).

[33] W. Kimura, IEEE Conference Record-Abstracts. The 31st IEEE International Conference on Plasma Science (ICOPS) (IEEE Service Center, Piscataway, NJ, 2004), p. 158.

[34] J. R. Marques, J.P. Geindre, F. Amiranoff, P. Audebert, J. C. Gauthier, A. Antonetti, and G. Grillon, Phys. Rev. Lett. 76, 3566 (1996). 